\title{
Identification and characterization of GMMYB118 responses to drought and salt stress
}

Yong-Tao Du ${ }^{1 \dagger}$, Meng-Jie Zhao ${ }^{1 \dagger}$, Chang-Tao Wang ${ }^{2}$, Yuan Gao ${ }^{1}$, Yan-Xia Wang ${ }^{3}$, Yong-Wei Liư ${ }^{4}$, Ming Chen ${ }^{1}$, Jun Chen ${ }^{1}$, Yong-Bin Zhou', Zhao-Shi Xu ${ }^{1 *}$ (D) and You-Zhi Ma'

\begin{abstract}
Background: Abiotic stress severely influences plant growth and development. MYB transcription factors (TFs), which compose one of the largest TF families, play an important role in abiotic stress responses.

Result: We identified 139 soybean MYB-related genes; these genes were divided into six groups based on their conserved domain and were distributed among 20 chromosomes (Chrs). Quantitative real-time PCR (qRT-PCR) indicated that GmMYB118 highly responsive to drought, salt and high temperature stress; thus, this gene was selected for further analysis. Subcellular localization revealed that the GmMYB118 protein located in the nucleus. Ectopic expression (EX) of GmMYB118 increased tolerance to drought and salt stress and regulated the expression of several stress-associated genes in transgenic Arabidopsis plants. Similarly, GmMYB118-overexpressing (OE) soybean plants generated via Agrobacterium rhizogenes (A. rhizogenes)-mediated transformation of the hairy roots showed improved drought and salt tolerance. Furthermore, compared with the control (CK) plants, the clustered, regularly interspaced, short palindromic repeat (CRISPR)-transformed plants exhibited reduced drought and salt tolerance. The contents of proline and chlorophyll in the OE plants were significantly greater than those in the CK plants, whose contents were greater than those in the CRISPR plants under drought and salt stress conditions. In contrast, the reactive oxygen species (ROS) and malondialdehyde (MDA) contents were significantly lower in the OE plants than in the CK plants, whose contents were lower than those in the CRISPR plants under stress conditions.
\end{abstract}

Conclusions: These results indicated that GmMYB118 could improve tolerance to drought and salt stress by promoting expression of stress-associated genes and regulating osmotic and oxidizing substances to maintain cell homeostasis.

Keywords: MYB transcription factor, Genome-wide analysis, Drought tolerance, Salt tolerance, CRISPR, Soybean

\section{Background}

Drought, salt and temperature stresses severely affect plant growth and agricultural production, threatening the survival of plants. Under stressful conditions, transcriptomic changes were the earliest responses in plants [1]. Gene expression analyses in plants have revealed that stress-responsive genes can be divided into two categories: effector genes and regulatory genes [2]. The products of

\footnotetext{
* Correspondence: xuzhaoshi@caas.cn

${ }^{\dagger}$ Yong-Tao Du and Meng-Jie Zhao contributed equally to this work. 'Institute of Crop Sciences, Chinese Academy of Agricultural Sciences (CAAS)/National Key Facility for Crop Gene Resources and Genetic Improvement, Key Laboratory of Biology and Genetic Improvement of Triticeae Crops, Ministry of Agriculture, Beijing 100081, China

Full list of author information is available at the end of the article
}

regulatory genes, which include membrane-localized receptors, calcium sensors, kinases and transcription factors (TFs), participate in further signal transduction regulation and gene expression [1]. TFs regulate gene expression by specifically binding to the cis-acting elements of downstream genes to influence many important cellular processes, such as signal transduction, morphogenesis and environmental stress responses $[3,4]$.

Based on the characteristics of their DNA-binding domain (DBD), TFs were divided into different families, such as bZIP, MYB, NAC, ERF, WRKY and AP2 families [5-9]. The MYB TFs, which represent the largest family in plants, can be divided into different subfamilies depending on the number of adjacent

(c) The Author(s). 2018 Open Access This article is distributed under the terms of the Creative Commons Attribution 4.0 International License (http://creativecommons.org/licenses/by/4.0/), which permits unrestricted use, distribution, and reproduction in any medium, provided you give appropriate credit to the original author(s) and the source, provide a link to the Creative Commons license, and indicate if changes were made. The Creative Commons Public Domain Dedication waiver (http://creativecommons.org/publicdomain/zero/1.0/) applies to the data made available in this article, unless otherwise stated. 
repeats within the MYB domain. Each repeat forms a helix-turn-helix structure of approximately 53 amino acids [10]. MYB-like proteins with one repeat were considered MYB-related (containing a single or a partial MYB repeat), those with two were regarded as R2R3-type MYBs (2R-MYB), those with three were regarded as R1R2R3 -type MYBs (3R-MYBs), and those with four repeats were regarded as 4 R-MYBs $[5,9,11-15]$.

The majority of MYB TFs, especially R2R3-MYBs, play important roles in response to abiotic stresses [6, 16-19]. For example, Chen identified 30 MYB genes that respond to multiple abiotic stresses in peanut [19]. TaMYB80 improved tolerance to high temperature and drought in wheat [6]. TaMYB56-B enhanced tolerance to freezing and salt stresses in transgenic Arabidopsis [16]. Compared to R2R3-MYB TFs, the MYB-related genes were mainly characterized for their role in processes, such as the control of cellular morphogenesis, flavonoid biosynthesis, hypocotyl elongation and circadian rhythm [20-24]. AtWER was an early regulator of epidermal cell fate in the root and hypocotyl [21]. Ammixta participated in the transcriptional control of epidermal cell shape [22]. Yi et al. reported that an R1 MYB transcription factor, GmMYB176, regulates GmCHS8 expression and isoflavonoid synthesis in soybean [25]. However, there were few reports that the MYB-related gene involved in abiotic stresses $[12,26]$. It is important to make clear whether more MYB-related genes participate in abiotic stresses.

Soybean (Glycine max) is widely cultivated and is one of the most important cash crops because of its high protein and oil content. However, its growth and grain yield are severely affected by drought and salt stresses. In some crops, different MYB TFs were characterized by their support of specific roles in response to water deficit and salt stress $[6$, 13, 17, 19]. Despite the whole genome of soybean being sequenced years ago [27], few studies have investigated the MYB-related TFs in this species. In this study, we provided a list of MYB-related family members based on soybean genome sequencing. Further investigation revealed that a MYB-related gene, GmMYB118, was significantly regulated by salt and drought treatment, and overexpression of GmMYB118 improved tolerance to drought and salt in both Arabidopsis and soybean. In contrast, the transformed plants of GmMYB118 via the clustered, regularly interspaced, short palindromic repeat (CRISPR) system exhibited reduced drought and salt tolerance. Our study provides a foundation for understanding the functions of the GmMYB118 gene in abiotic stress responses.

\section{Results}

\section{Identification and chromosomal distribution of soybean} MYB-related genes

The species of MYB-related TF genes were various in different species (Table 1). To analyze the entire MYB-related
Table 1 Numbers of MYB-related TFs in different species

\begin{tabular}{lll}
\hline Species & Number & Reference \\
\hline Arabidopsis thaliana & 68 & Du et al, 2013 [28] \\
Arachis hypogaea & 20 & Chen et al, 2014 [19] \\
Oryza sativa & 70 & Dubos et al, 2010 [14] \\
Zea mays & 72 & Du et al, 2013 [28] \\
Glycine max & 127 & Du et al, 2013 [28] \\
\hline
\end{tabular}

family in soybean, we queried several databases such as Phytozome, TFDB, Pfam, SMART and ScanProsite [10]. Previous work revealed 127 MYB-related TF genes in soybean [28]; and these genes were searched against the above websites. After deleting redundant sequences and screening typical MYB-related domains, we identified 139 genes in soybean. All the MYB-related genes located on twenty chromosomes (Chrs) by using MapInspect software. Chrs 4 and 6 of soybean contain many MYB-related genes-approximately $14.8 \%$, while fewer numbers of MYB-related genes located on Chrs 7 and 20. Chrs 5 to 9 presented a relatively uniform distribution (Fig. 1a). As shown in Fig. 1b, the MYB-related genes tended to be distributed on both arms of Chrs 9, 10, 11, 12, 15, 16, 17 and 18. On the other Chrs, the MYB-related genes were evenly distributed (Chrs 7,8 and 13) or were abundantly distributed at either end.

\section{Phylogenetic tree analysis with amino acid sequence of 139 MYB-related genes}

Alignment of the amino acid sequences was used to construct a phylogenetic tree by MEGA 6 via the neighbor-joining (NJ) method. As shown in Fig. 2, the phylogenetic tree was divided into 5 groups (I-V). The sequence $\mathrm{SHAQK}(\mathrm{Y} / \mathrm{F}) \mathrm{F}$ was highly conserved in group I. Group II shared a consistent DLKDKW sequence. For other groups, although these MYB proteins have no conserved domain, they have conserved amino acid sites. The high bootstrap values for the node supported that the other members of 139 MYB proteins were clustered in three groups (III, IV and V), respectively.

\section{Screening candidate genes for further analysis}

According to the gene accession number we submitted to the soybase website (http://soybase.org/soyseq/) [10], we obtained the tissue expression data of quantified prediction for a diverse set of fourteen tissue types (Additional file 1: Figure S1). It showed that the expression level of several genes in roots, leaf nodes, leaves and flowers was higher than that in seeds and pods. It may suggest that these genes play a crucial role in soybean growth and development. For further analysis, we screened 10 members from the 139 MYB genes that according to the amount of expression level more than 300 from soybase website prediction in root, including GmMYB7/20/31/49/75/81/92/105/110/118 (Fig. 3a). It 

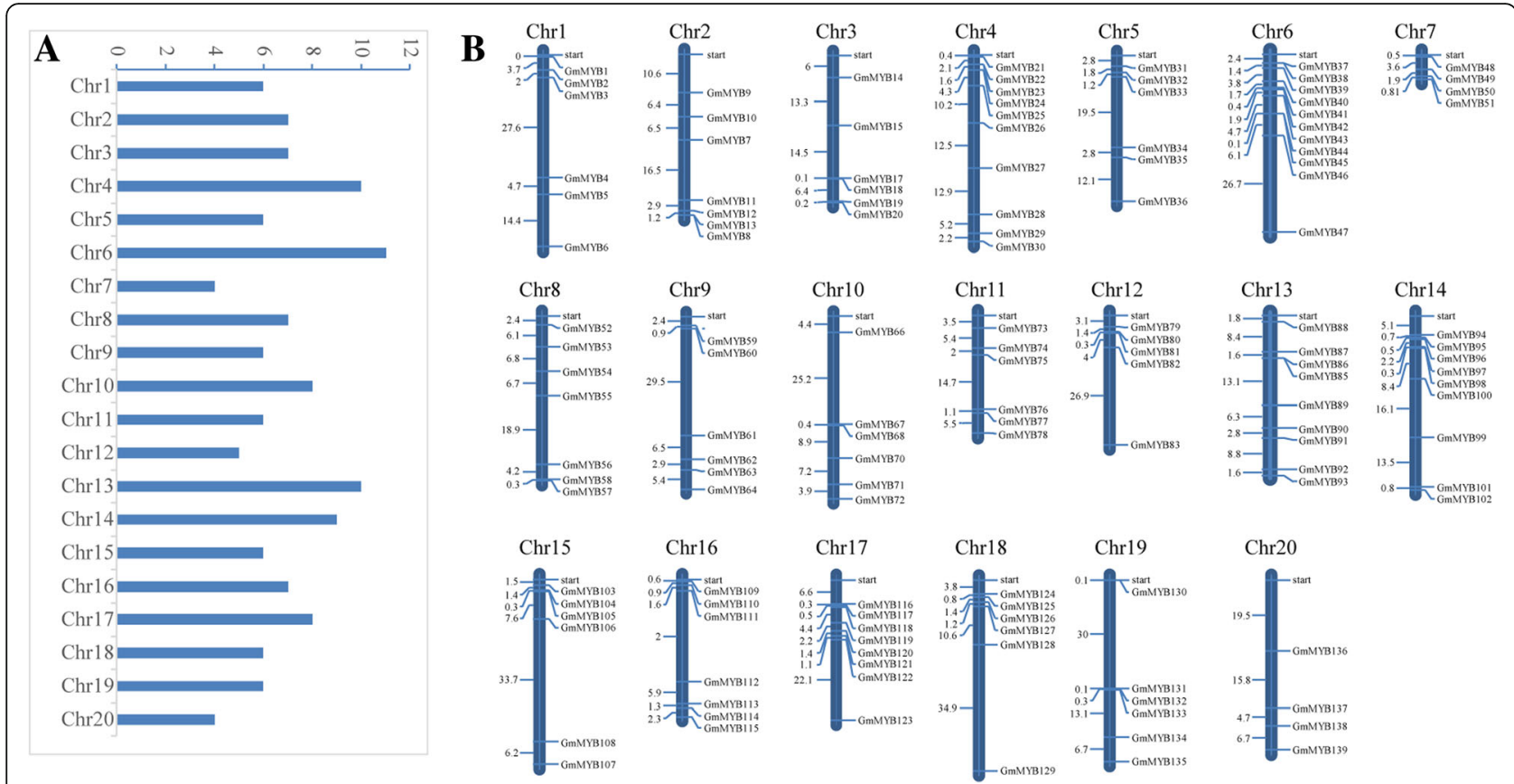

Fig. 1 Chromosomal distribution of 139 MYB-related genes in soybean. We identified 139 MYB-related genes in soybean by researching several databases such as Phytozome, TFDB, Pfam, SMART and ScanProsite. The members of MYB-related genes were distributed on different Chr (numbers 1-20) (a). The physical location of each member was shown in Figure (b). The deep blue bars represent the Chrs, and the Chr numbers were shown on the top of the bars. The length of the bar was not represented the size of the Chr. The numbers on the left side of the bars show the distances in megabases $(\mathrm{Mb})$ between neighboring genes

may suggest that these genes play an important role in soybean roots.

\section{Gene structure analysis of the ten selected MYB-related TFs}

To characterize the ten select MYB-related genes, we analyzed their structure using Gene Structure Display Server (http://gsds.cbi.pku.edu.cn/) by submitting coding DNA sequences (CDS) and genomic sequences, and we retrieved basic information (Table 2). As shown in Fig. $3 \mathrm{~b}$, the ten MYB-related genes presented with an exon-intron structure. The results showed that the MYB-related genes tended to have closer genetic relationships with more similar structures. For example, GmMYB7/31/118, GmMYB75/92/105 and GmMYB20/ $31 / 110$ exhibit similar gene structures, which suggests that they evolved from the same pattern.

\section{Promoter regions of the ten MYB-related genes contain various stress-responsive elements}

The 2000 bp region upstream of the ATG start codon in the promoters of the ten MYB-related genes was selected. To investigate the mechanism involved in the response to abiotic stresses, plant cis-acting elements and PLACE (http://bioinformatics.psb.ugent.be/webtools/plantcare/html/) were used to analyze the regions of the ten gene promoters. A number of regulatory elements that respond to drought and salt stress were identified, including ABRE (ABA-induced), DRE (drought-induced), GT-1 (salt-induced), MYB (drought) and MYC (drought and cold) elements. In addition, the numbers of cis-elements for MYB, MYC and GT-1 TFs were greater than other cis-elements in these promoters of the ten genes (Table 3). This information revealed that the ten MYB-related genes may be involved in abiotic stress responses, such as drought, salt and cold responses.

\section{Several candidates are involved in multiple abiotic stresses}

To gain insight into potential functions, we initially examined the expression patterns of the ten MYB-related genes in response to various abiotic stresses by quantitative real-time PCR (qRT-PCR) (Fig. 4). Under drought treatment, the expression of $G m M Y B 20 / 31 / 118$ increased by 2.42, 3.98 and 3.11 -fold at 1,5 and $5 \mathrm{~h}$, respectively, the transcription levels of other genes did not change significantly (A). For salt treatment, the expression peaks of GmMYB7/31/118 occurred at 5, 5 and $12 \mathrm{~h}$, respectively, which were equivalent to $6.45,6.06$ and 6.54-fold increases, respectively. The expression of other genes did not change significantly (B). Under heat treatment, the expression of $G m M Y B 7 / 31 / 75 / 118$ increased by 3.41, 1.96, 1.89 and 2.40 -fold at $5 \mathrm{~h}$, respectively, the expression levels 


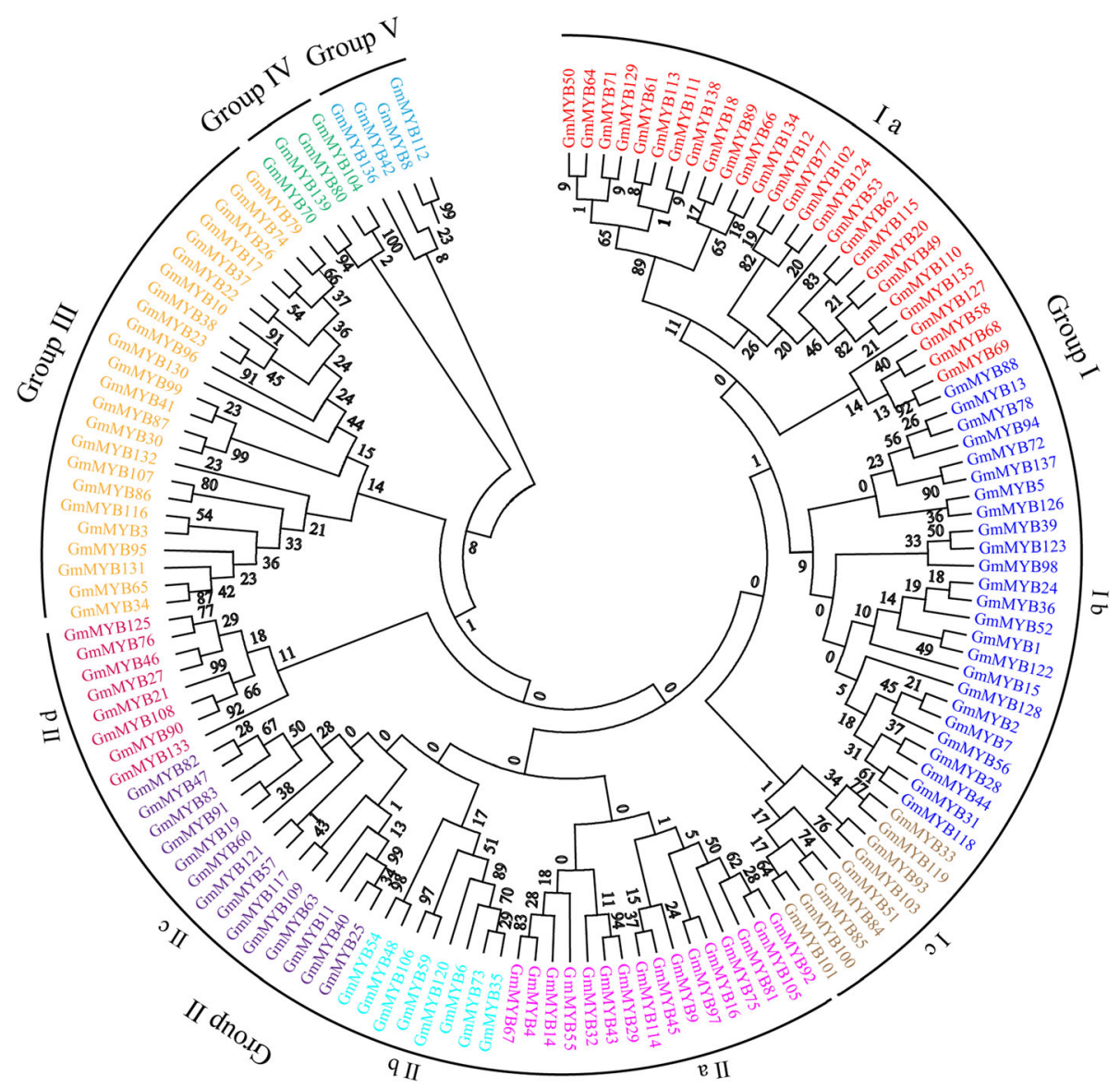

Fig. 2 Phylogenetic tree of the MYB-related TFs subfamily in soybean. Amino acid sequences were aligned via ClustalX and were manually corrected. The phylogenetic tree was constructed with MEGA 6 in conjunction with the NJ method. The same color represents the same group

of other genes did not change significantly $(C)$. Under cold treatment, the accumulation of GmMYB20/49/110/118 transcripts increased gradually and peaked at 1, 5, 5 and $12 \mathrm{~h}$; however, the accumulation of GmMYB118 transcript level was rapidly decreased, it was similar to the CK plants at $12 \mathrm{~h}$. The highest levels of GmMYB20/49/110/118 were equivalent to 4.16, 7.6, 6.05 and 5.2-fold increases, respectively (D). These results indicated that the accumulation of transcript levels of GmMYB7/20/31/118 was affected by various abiotic stresses. Among those genes, GmMYB118 clearly responded to multiple abiotic stresses, including drought, salt, heat and cold (Fig. 4). For this reason, GmMYB118 was selected for further investigation.

\section{Subcellular localization of GmMYB118 in Arabidopsis}

To determine the subcellular localization of GmMYB118, GmMYB118 was fused to the N-terminus of the humanized green fluorescent protein (hGFP) reporter gene and ligated into an 16318hGFP expression vector under control of the cauliflower mosaic virus (CaMV) 35S promoter. The cDNA coding sequences of AtWRKY25 (At2g30250) that Located in the nucleus [29] were fused to the $\mathrm{N}$-terminus of the RFP gene under the control of the CaMV 35S promoter. Subcellular localization of GFP and RFP expression in Arabidopsis mesophyll protoplasts was observed after cotransformation. The GmMYB118::hGFP fusion protein localized in the nucleus (Additional file 1: Figure S2A). These observations suggested that GmMYB118 could enter the nucleus to function.

\section{GmMYB118 provided drought tolerance in Arabidopsis}

Overexpression of stress-inducible genes in plants represents an effective strategy for improving abiotic stress tolerance [3, 4, 30-32]. To further investigate the biological functions of the GmMYB118 gene, three T3 Ectopic expression (EX) lines were selected for analysis under polyethylene glycol (PEG6000) treatment to simulate drought stress. Before conducting the experiment, three-week-old Arabidopsis seedlings were subjected to qRT-PCR analysis of GmMYB118 gene expression in ectopic expression and wild type (WT) plants (Additional file 1: Figure S2B). Expression of AtActin was analyzed as a loading control 


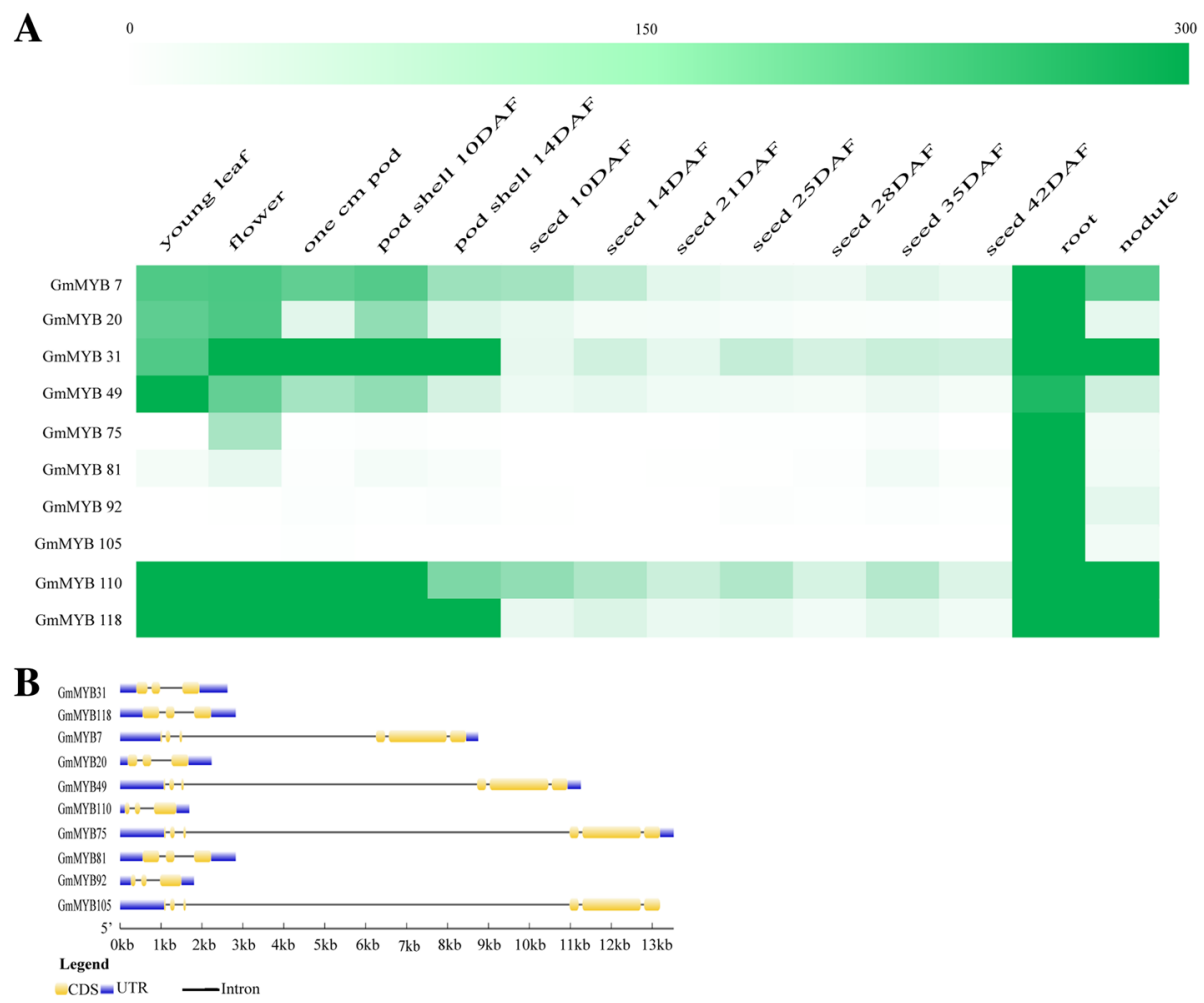

Fig. 3 Quantified prediction of tissue expression in soybean and sequence conservation analysis of the ten selected MYB-related TF genes. In accordance with the quantified prediction of fourteen tissue expression provided by SoyBase, the ten MYB-related TF genes that according to the quantified expression level of more than 300 were screened from the 139 MYB-related genes for further analysis. The deeper color represents a greater quantity (a). We analyzed the structure using Gene Structure Display Server (http://gsds.cbi.pku.edu.cn/) by submitting CDSs and genomic sequences $(\mathbf{b})$

(Additional file 1: Table S1). The relative expression level of GmMYB118 was equivalent to 8 12 fold in Arabidopsis.

For germination assays, seeds of EX and WT lines were germinated on 1/2-strength Murashige and Skoog (MS) media containing various concentrations of PEG6000, and the germination rates was determined at $0,12,24,36,48,60$ and $72 \mathrm{~h}$. All lines exhibited similar germination rates on $1 / 2$-strength MS media. However, in the presence of PEG6000, the germination of the EX seeds was inhibited, and the degree of inhibition was greater than that of the WT seeds (Additional file 1: Figure S3A). Under normal condition, the germination rate of the WT and EX seeds was about 94 96\% at the time points of $72 \mathrm{~h}$ (Additional file 1: Figure S3B). Under 3\% PEG6000 treatment, the germination rate of the EX seeds was 64.06 72.91\%, which was lower than that of the WT seeds $(81.77 \%)$ at the time points of $24 \mathrm{~h}$ (Additional

Table 2 Basic information concerning ten MYB-related genes in soybean

\begin{tabular}{|c|c|c|c|c|c|c|}
\hline Gene & Gene ID number & Amino acids & $\mathrm{p} /$ & Molecular mass (kD) & Chromosome & Domain location \\
\hline GmMYBT & Glyma02g03020 & 300 & 10.41 & 32.16 & 2 & $94-138$ \\
\hline GmMYB20 & Glyma03g42260 & 748 & 6.15 & 82.07 & 3 & $24-68$ \\
\hline GmMYB31 & Glyma05g01640 & 285 & 9.66 & 31 & 5 & $80-124$ \\
\hline GmMYB49 & Glyma07g05410 & 750 & 6.55 & 82.3 & 7 & $24-68$ \\
\hline GmMYB75 & Glyma11g15180 & 204 & 4.48 & 22.8 & 11 & $8-62,68-113$ \\
\hline GmMYB81 & Glyma12g07110 & 750 & 6.2 & 82.4 & 12 & $8-62,68-113$ \\
\hline GmMYB92 & Glyma13g40830 & 350 & 9.09 & 38.12 & 13 & $8-55,61-106$ \\
\hline GmMYB105 & Glyma15g04620 & 192 & 4.61 & 21.5 & 15 & $8-55,61-106$ \\
\hline GmMYB110 & Glyma16g01980 & 194 & 5.07 & 22.13 & 16 & $24-68$ \\
\hline GmMYB118 & Glyma17g10250 & 194 & 4.79 & 22.14 & 17 & $144-188$ \\
\hline
\end{tabular}


Table 3 Distribution of cis-acting elements within ten MYBrelated gene promoters in soybean

\begin{tabular}{lllllllll}
\hline Gene & ABRE & DRE & ERE & GARE & GT-1 & LTRE & MYB & MYC \\
\hline GmMYB7 & 9 & 1 & 0 & 1 & 43 & 0 & 14 & 10 \\
GmMYB20 & 7 & 0 & 0 & 0 & 23 & 1 & 16 & 20 \\
GmMYB31 & 1 & 0 & 0 & 0 & 25 & 0 & 15 & 20 \\
GmMYB49 & 16 & 0 & 1 & 0 & 34 & 1 & 20 & 14 \\
GmMYB75 & 1 & 4 & 1 & 4 & 28 & 7 & 21 & 24 \\
GmMYB81 & 0 & 3 & 0 & 3 & 27 & 5 & 10 & 14 \\
GmMYB92 & 7 & 0 & 3 & 1 & 34 & 1 & 20 & 24 \\
GmMYB105 & 6 & 0 & 5 & 2 & 27 & 1 & 20 & 22 \\
GmMYB110 & 12 & 2 & 1 & 1 & 52 & 3 & 24 & 24 \\
GmMYB118 & 3 & 4 & 0 & 3 & 34 & 3 & 16 & 22 \\
\hline
\end{tabular}

file 1: Figure S3C). Under 6\% PEG6000 treatment, the germination rate of the EX seeds was $33.85 \sim 34.89 \%$, which was lower than that of the WT seeds $63.02 \%$ at the time points of $24 \mathrm{~h}$ (Additional file 1: Figure S3D). Under 9\% PEG6000 treatment, the germination rate of the EX seeds was 76.56 81.77\%, which was lower than that of the WT seeds (94.79\%) at the time points of $48 \mathrm{~h}$ (Additional file 1: Figure S3E).

For phenotyping of seedlings, the six-day-old Arabidopsis seedlings were transferred to 1/2-strength MS medium contained different concentrations of PEG6000 for 7 days. The phenotypes of the transgenic seedlings were similar to those of the WT seedlings under normal conditions (Fig. 5a). As shown in Fig. 5, PEG6000 treatment reduced the root growth of both EX and WT seedlings to some extent (Fig. 5b-d). Under 3 and 9\% PEG6000 treatments, the root lengths of the GmMYB118 lines were 11.86 13.65 cm and 9.34 10.39 $\mathrm{cm}$, respectively, which were significantly longer than those of WT lines $(8.38 \mathrm{~cm}$ and $6.36 \mathrm{~cm}$, respectively) (Fig. 5f, h). The root length of WT seedlings was also shorter than that EX seedlings under 6\% PEG6000 treatment (Fig. 5g). In addition, at the later seedling stage, three-week-old EX and WT seedlings were not watered for 14 days, after which they were pictured after being rewatering 3 days (Fig. 7a). The survival rate of the

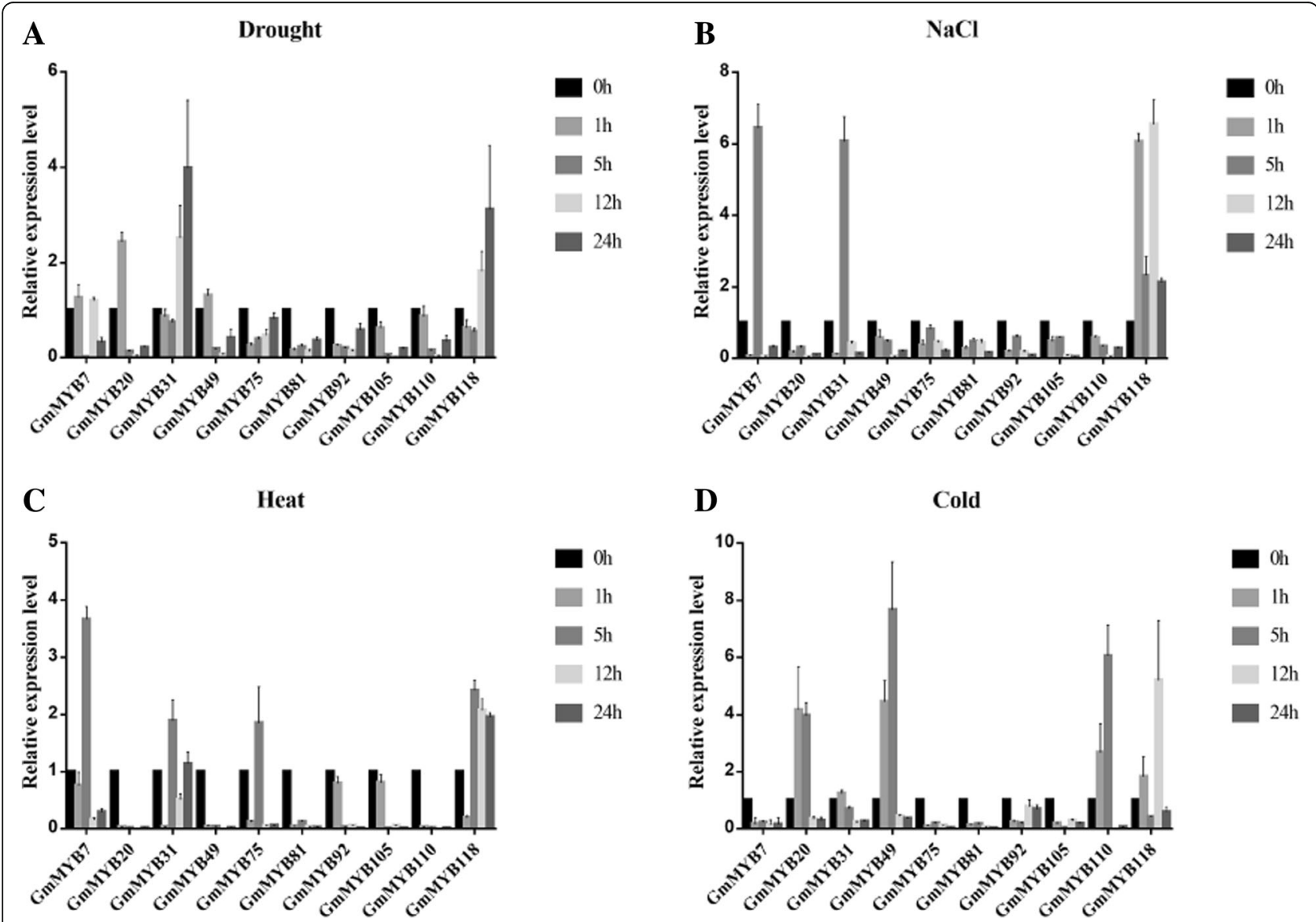

Fig. 4 Expression patterns of the ten selected MYB-related TF genes under salt, drought, cold and heat treatment. Fifteen-year-old seedling of soybean were treated with Drought (a), $\mathrm{NaCl}(\mathbf{b})$, Heat (c) and Cold (d) for 0, 1, 5, 12 and $24 \mathrm{~h}$. The expression patterns of the ten select MYBrelated TF genes under various abiotic stresses were quantified by qRT-PCR analysis. GMMYB118 clearly responded to multiple abiotic stresses including drought, salt, cold and heat stresses (a-d). The data were shown as the means \pm SDs of three experiments 


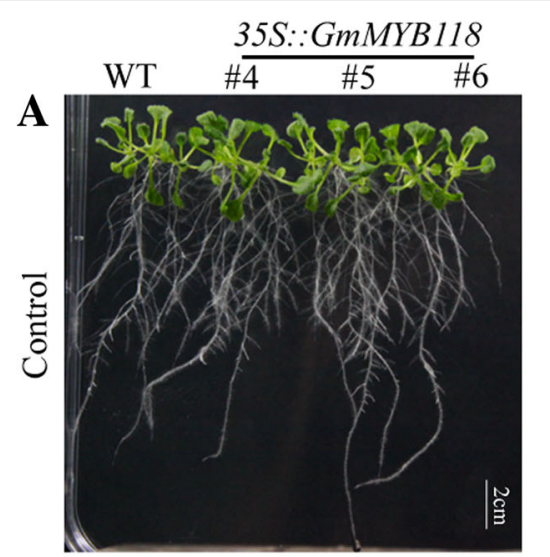

E

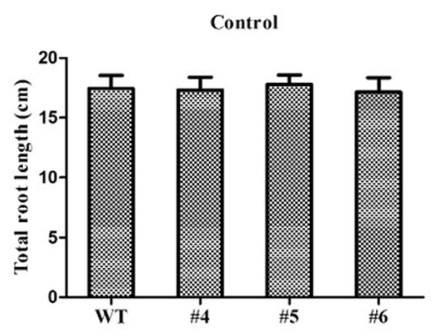

B

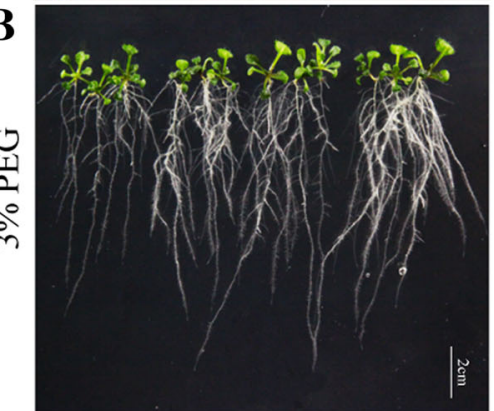

F
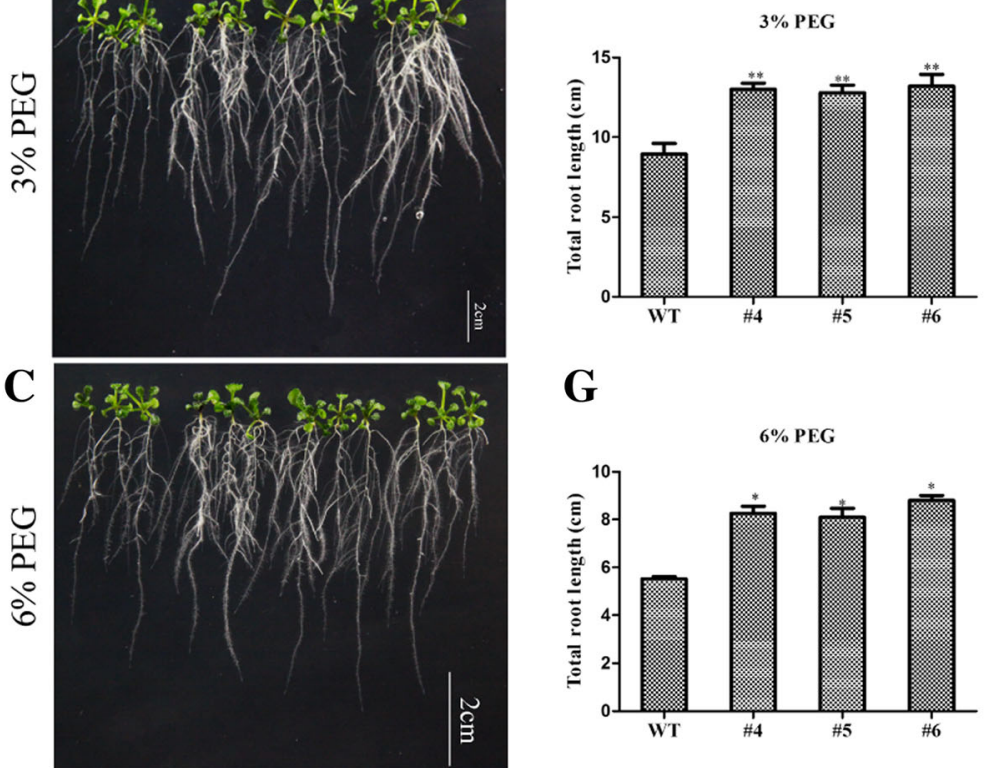

G

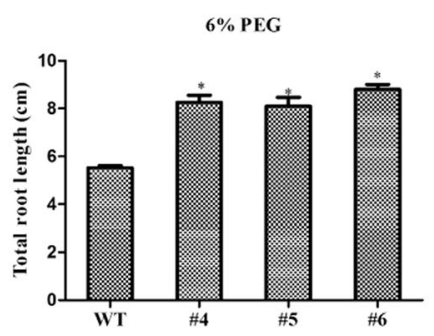

D

H
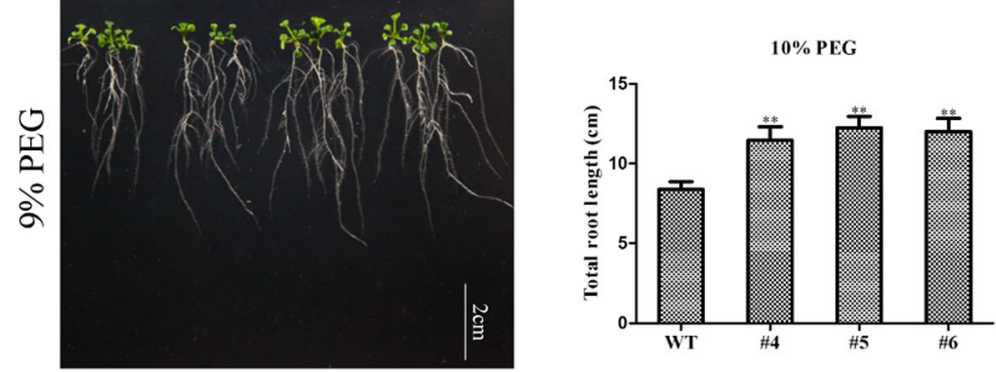

Fig. 5 Root length phenotypes of EX lines under PEG treatment. The six-day-old seedlings grown on 1/2 MS were transferred to 1/2 MS medium containing different concentrations of PEG6000. A week later, the growth of roots was photographed of EX and WT (Col-0) seedlings under 0, 3, 6 and 9\% PEG6000 treatment (a-d). Compared with those of the WT (Col-0) seedlings, the statistical results of total root length were shown of the EX seedlings under 0, 3, 6 and 9\% PEG treatment (e-h). The data were shown as the means \pm SDs $(n=30)$ of three experiments. ANOVA test demonstrated that there were significant differences ( $* 0.05$, ${ }^{* *} P<0.01$ )

EX lines after being rewatering 3 days was 90.05 95.63\%, which was significantly higher than that of the WT lines (40.50\%) (Fig. 7c). These results suggest that $G m M Y B 118$ may potentially function to increase the tolerance of the transgenic plants to drought stress.

\section{GmMYB118 provided salt tolerance in Arabidopsis}

To elucidate the role of the GmMYB118 in plant growth and development under high salt conditions, salt tolerance experiments involving transgenic and WT lines were carried out. For germination assays, seeds of EX 
and WT lines were germinated on 1/2-strength MS media that contained various concentrations of $\mathrm{NaCl}$, and the germination rates was determined at $0,12,24$, 36, 48, 60 and $72 \mathrm{~h}$. Both EX and WT seeds exhibited similar germination rates on 1/2-strength MS media without $\mathrm{NaCl}$ (Additional file 1: Figure S4B). In the presence of $\mathrm{NaCl}$, the germination of both the EX and WT seeds was inhibited (Additional file 1: Figure S4A). Under $75 \mathrm{mM} \mathrm{NaCl}$ treatment, the germination rate of the EX seeds was 30.18 35.37\%, which was lower than that of the WT seeds (55.23\%) at the time points of $48 \mathrm{~h}$ (Additional file 1: Figure S4C). Under $100 \mathrm{mM} \mathrm{NaCl}$ treatment, the germination rate of the EX seeds was $50.47 \sim 53.29 \%$, which was lower than that of the WT seeds $(69.52 \%)$ at the time points of $48 \mathrm{~h}$ (Additional file 1: Figure S4D). Under $125 \mathrm{mM} \mathrm{NaCl}$ treatment, the inhibition of germination was more severe for the EX seeds than for the WT seeds. The germination rate of the EX seeds ranged from 14.28 18.86\%, which was lower than that of the WT seeds (47.62\%) (Additional file 1: Figure S4E).

For phenotyping, transgenic and WT Arabidopsis seeds were grown on $1 / 2 \mathrm{MS}$ media for 6 days at $22^{\circ} \mathrm{C}$, after which they were transferred to $1 / 2$-strength MS media that contained various concentrations of $\mathrm{NaCl}$ and grown for 7 days. The phenotypes of the EX seedlings were similar to those of the WT seedlings under normal conditions (Fig. 6a). As is shown in Fig. 6, under 75, 100 and $125 \mathrm{mM}$ $\mathrm{NaCl}$ treatments, the root length of the EX lines was significantly longer than that of the WT lines (Fig. 6b-d). Under 75 and $125 \mathrm{mM} \mathrm{NaCl}$ treatments, the total root length of the WT lines $(9.39$ and $7.69 \mathrm{~cm})$, which was significantly shorter than that of the transgenic lines ranged from 13.11 15.51 and 9.86 10.80 cm (Fig. 6f, h). This difference is most definitive in response to the $100 \mathrm{mM} \mathrm{NaCl}$ treatment: the total root length of the EX lines ranged from $11.24 \sim 13.51 \mathrm{~cm}$, which was significantly greater than that of the WT lines $(8.37 \mathrm{~cm})$ (Fig. $6 \mathrm{~g}$ ). Moreover, at the later seedling stage, three-week-old EX and WT seedlings were grown under $250 \mathrm{mM} \mathrm{NaCl}$ for 14 days; their phenotypes are shown in Fig. 7b. The survival rate of the EX lines ranged from $88.32 \sim 92.16 \%$, which was significantly greater than that of the WT liens (68.94\%) (Fig. 7d). Overall, these results suggested that GmMYB118 may be used to improve tolerance to salt stress in transgenic plants.

\section{GmMYB118 activated stress-responsive genes in Arabidopsis}

To elucidate the possible molecular mechanisms of the involvement of GmMYB118 in stress responses, the expression of drought- and salt-responsive marker genes including AtP5CS1 [33], AtDREB2A [34], AtCOR47 [30], AtCOR15A [4], AtRD29A [35], AtKIN1 [36], AtKIN2 [37], AtRD22 [38], AtRAB18 [39], AtADH1 [40], and
AtNCED3 [41] was investigated in EX lines. A 2-fold change in expression was arbitrarily considered to represent positive expression induction.

qRT-PCR analysis revealed no significant differences at the levels of expression of AtCOR47, AtDREB2A, AtKIN1, AtKIN2, AtRD29A and AtCOR15 between the EX lines and WT lines under normal conditions (Fig. 8a-f). Under drought conditions, the expression of these genes in the EX lines significantly higher than that in the WT lines (Fig. 8a-f), although the expression levels of AtP5CS1 and AtRAB18 did not differ (data not shown). On the other hand, compared with that in the WT lines, the expression levels of AtADH1, AtNCED3, AtCOR15 and AtRD29A in the EX lines significantly increased under salt conditions (Fig. $8 \mathrm{~g}-\mathrm{j}$ ), but these levels did not markedly differ under normal conditions (Fig. 8g-j). The expression level of AtRD22 did not significantly differ between the EX lines and the WT lines in either normal or drought conditions (data not shown). These results indicated that overexpression of GmMYB118 may activate the expression of drought- or salt-responsive genes in Arabidopsis, improving the drought and salt stress tolerance of transgenic lines.

\section{Targeted mutagenesis in soybean hairy roots and GUS staining}

To further confirm the functions of the GmMYB118 gene in soybean, two constructs (pCAMBIA3301-GmMYB118 and pCas9-GmU6-sgRNA) were generated for overexpression and for gene editing analysis with the CRISPR-Cas9 system (OE and CRISPR constructs, respectively) into soybean hairy roots.

Because the vector of pCAMBIA3301 carries the $\beta$-glucuronidase (GUS) reporter gene, we examined the expression level of GUS in accordance with the protocol of a GUS histochemical assay kit to detect the transformation efficiency of the vector by Agrobacterium rhizogenes (A. rhizogenes)-mediated transformation. The transformation efficiency was approximately $50 \%$ (Additional file 1 : Figure S5A). It can be inferred from the results of GUS staining that about $50 \%$ of the roots of each $\mathrm{OE}$ and CRISPR plant were positive roots. To detect the targeted gene mutations in soybean hairy roots, genomic DNA was collected and extracted for further detection of the target gene mutations in the hairy roots. The target gene was amplified with specific primers and sequenced, and the results showed that some bases were replaced without any insertions or deletions (Additional file 1: Figure S5B). Our results shown that $10 \%$ of roots of the coding sequence of GmMYB118 was edited in each CRISPR plant. The amino acid $\left(\mathrm{I}_{17}, \mathrm{~L}_{18}, \mathrm{~F}_{19}\right)$ of GmMYB118 in 77.5\% CRISPR plants was changed, such as from $\mathrm{I}_{17}$ to $\mathrm{M}_{17}, \mathrm{~L}_{18}$ to $\mathrm{A}_{18}, \mathrm{~F}_{19}$ to $\mathrm{S}_{19}$. These findings indicated that the CRISPR-Cas9 system modified the gene during hairy root development. 


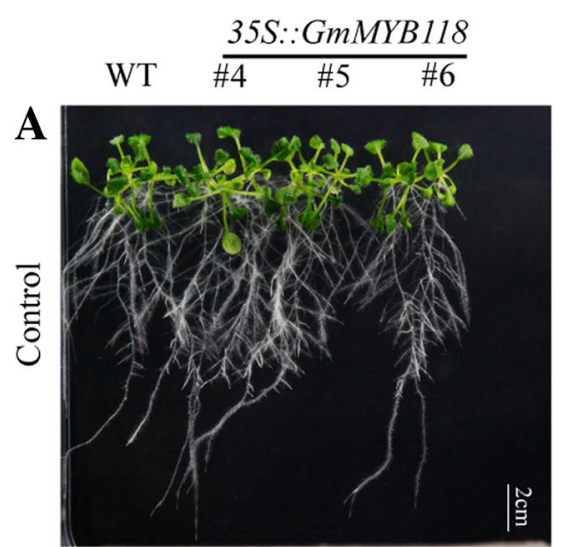

$\mathbf{E}$
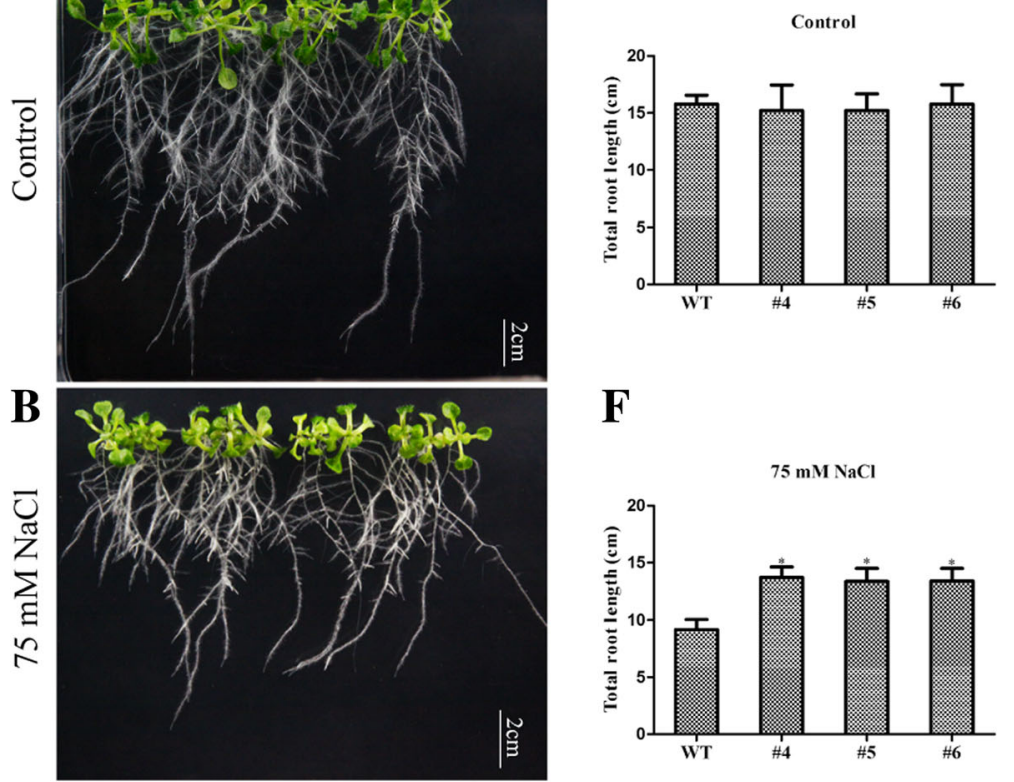

$\mathbf{F}$
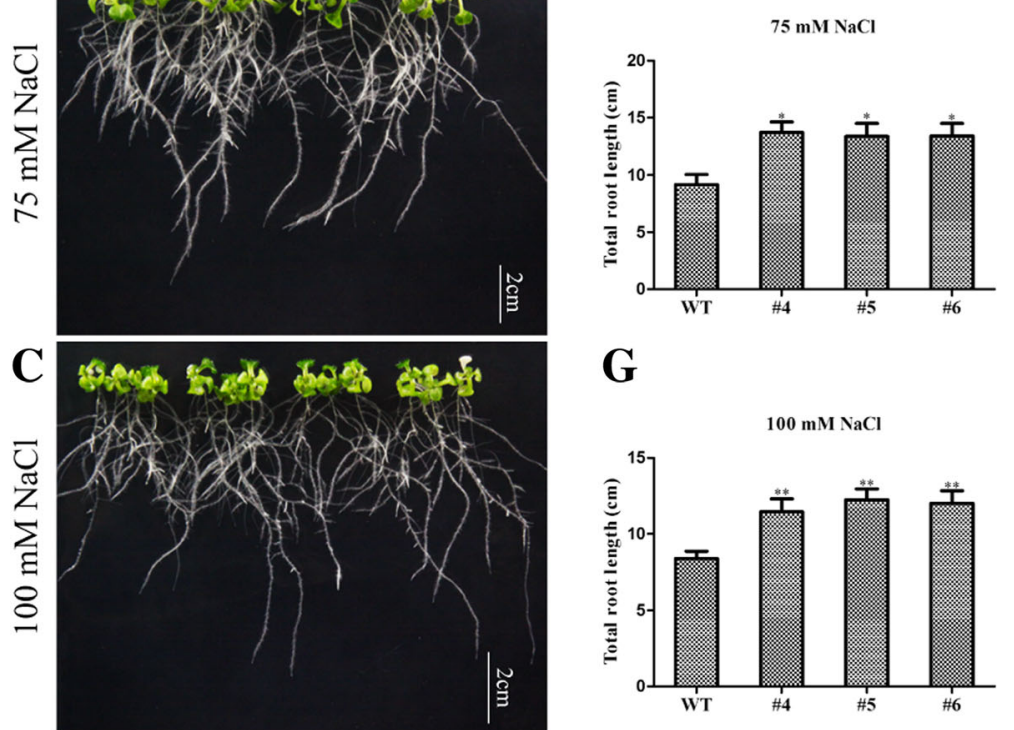

$\mathbf{G}$
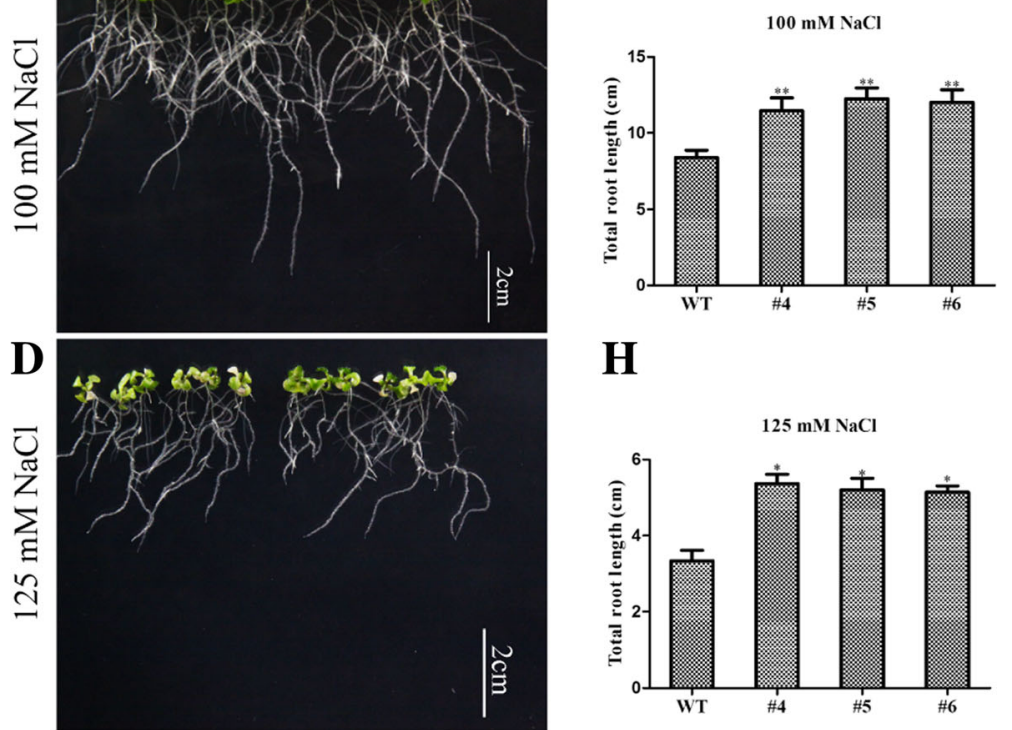

H

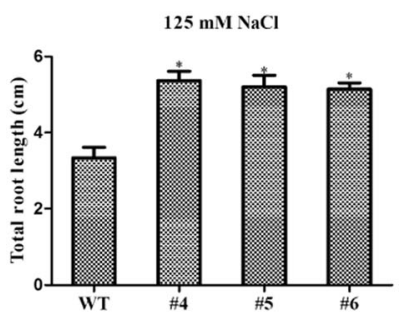

Fig. 6 Root length phenotypes of EX lines under $\mathrm{NaCl}$ treatment. The six-day-old seedlings grown on 1/2 MS were transferred to 1/2 MS medium containing different concentrations of $\mathrm{NaCl}$. A week later, the growth of roots was photographed of EX and WT (Col-0) seedlings under $0,75,100$ and $125 \mathrm{mM} \mathrm{NaCl}$ treatment (a-d). Compared with those of the WT seedlings, the statistical results of total root length are shown of the EX seedlings under $0,75,100$ and $125 \mathrm{mM} \mathrm{NaCl}$ treatment $(\mathbf{e}-\mathbf{h})$. The data were shown as the means \pm SDs $(n=30)$ of three experiments. ANOVA test demonstrated that there were significant differences $\left({ }^{*} P<0.05,{ }^{* *} P<0.01\right)$

\section{GmMYB118 improved drought and salt tolerance in transgenic soybean hairy roots}

The OE and CRISPR lines were analyzed for drought tolerance [1, 25, 42, 43]. For drought treatment, the hairy roots of the seedlings were not watered for 14 days, then rewatering for 3 days. The survival rate of the $\mathrm{OE}$ plants was $83.33 \%$, which was clearly greater that of the CK plants (33.33\%); however, the survival rate of the CRISPR plants was $16.67 \%$, which was worse than that of the CK plants (Fig. 9a). Similarly, the survival rate of the OE plants was $66.67 \%$ under salt conditions, which was clearly greater than that of the CK plants (48.33\%). 


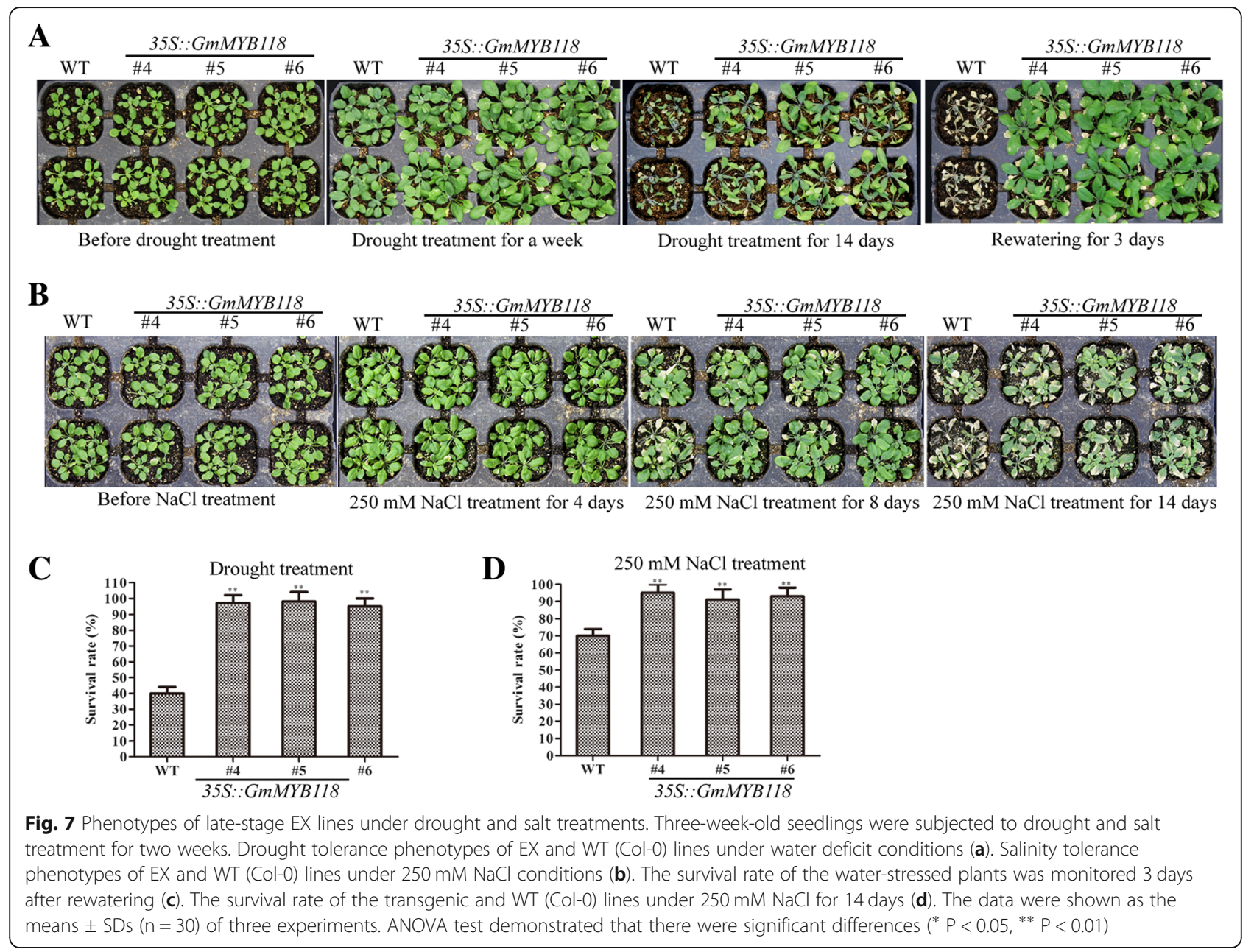

The survival rate of the CRISPR plants was $25.00 \%$ lower than that of the CK plants (Fig. 10a).

To investigate the potential physiological mechanism involved in improving the drought resistance of the $\mathrm{OE}$ lines, the proline, malondialdehyde (MDA) and chlorophyll contents in the OE, CK and CRISPR plants were measured under both normal growth and stress conditions. The stress condition was described in the method. The proline and chlorophyll contents were $86.41 \mu \mathrm{g} / \mathrm{g}$ and $0.65 \mathrm{mg} / \mathrm{g}$, respectively, which were significantly greater in the $\mathrm{OE}$ plants than in the CK plants $(47.16 \mu \mathrm{g} / \mathrm{g}$ and $0.39 \mathrm{mg} / \mathrm{g}$, respectively). The proline and chlorophyll contents in the CRISPR plants $(16.44 \mu \mathrm{g} / \mathrm{g}$ and $0.29 \mathrm{mg} / \mathrm{g}$, respectively) were evidently lower than those in the CK plants under drought conditions (Fig. 9b, c). Similarly, the proline and chlorophyll contents were $88.17 \mu \mathrm{g} / \mathrm{g}$ and $0.62 \mathrm{mg} / \mathrm{g}$ in the OE plants, respectively, and were significantly greater than those in the CK plants $(46.70 \mu \mathrm{g} / \mathrm{g}$ and $0.37 \mathrm{mg} / \mathrm{g}$, respectively). The same contents were evidently $12.45 \mu \mathrm{g} / \mathrm{g}$ and $0.20 \mathrm{mg} / \mathrm{g}$ lower in the CRISPR plants than in the CK plants under salt conditions, respectively (Fig. 10b, c). Under both drought and salt conditions, the MDA content in the OE plants was lower than that in both the CK and CRISPR plants (Figs. 9d and 10d). By contrast, the MDA contents among all plants did not differ under normal conditions (Figs. 9b-d and 10b-d).

We detected the expression of GmMYB118 in the hairy roots of transgenic plants subjected to drought and $\mathrm{NaCl}$ treatments. Compared with that in the CK plants, the expression in the OE plants increased by 7.9 times, while that of the CRISPR plants decreased by 2.3 times under $\mathrm{NaCl}$ treatment. The expression in the $\mathrm{OE}$ plants was 5 times greater than that in the CK plants, while the expression in the CRISPR plants was 2 times lower than that in the $\mathrm{CK}$ plants under drought treatment (Additional file 1: Figure S6).

\section{Overexpression of $\mathrm{GmMYB} 118$ reduced the concentration of $\mathrm{O}_{2}^{-}$and $\mathrm{H}_{2} \mathrm{O}_{2}$}

Because stress and the intracellular reactive oxygen species (ROS) content affect plant growth and development, we stained soybean leaves with 3,3-diaminobenzidine (DAB) and nitroblue tetrazolium (NBT) to detect $\mathrm{H}_{2} \mathrm{O}_{2}$ 

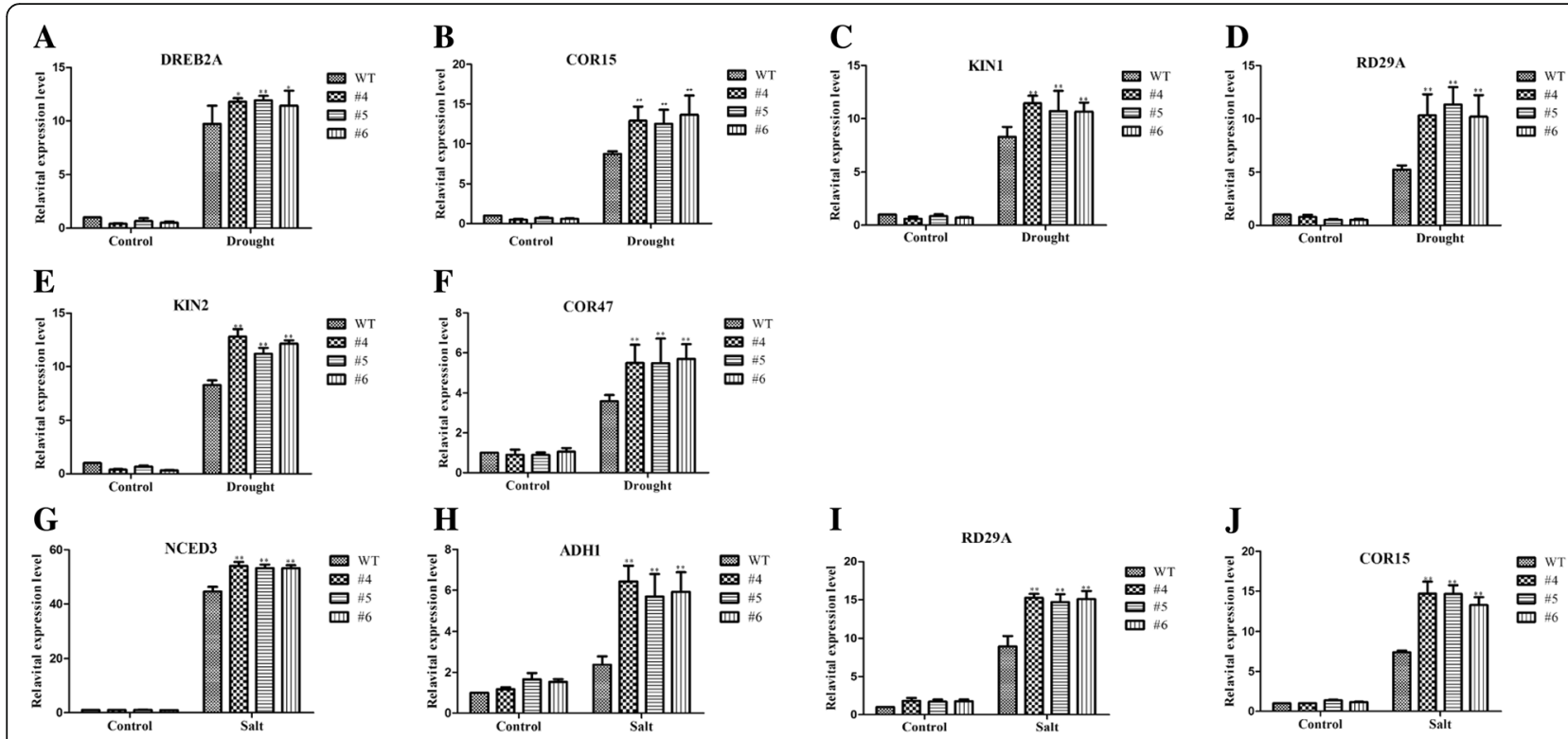

Fig. 8 GMMYB118 regulates stress-responsive gene expression in transgenic Arabidopsis plants. Extraction of RNA from two-week-old seedlings grown on 1/2 MS medium with drought and $\mathrm{NaCl}(100 \mathrm{mM})$ treatment for $2 \mathrm{~h}$. Gene expression level was quantified by qRT-PCR assays. Expression of AtActin was analyzed as a control. Gene-specific primers were used to detect the expression levels of stress-related genes. The expression levels of drought-related genes significantly increased in transgenic Arabidopsis plants under drought treatment (a-f). The expression levels of salt-related genes significantly increased in the transgenic Arabidopsis plants under salt treatment $(\mathbf{g}-\mathbf{j})$. The data were the means \pm SDs of three experiments. ANOVA test demonstrated that there were significant differences ( ${ }^{*} P<0.05,{ }^{* *} P<0.01$ )

and $\mathrm{O}_{2}{ }^{-}$contents under normal or stress conditions in OE, CK and CRISPR plants. The stress condition was described in the method. Under normal growth conditions, the DAB and NBT staining of all plant leaves showed no differences (Figs. 9f-g and $10 \mathrm{f}-\mathrm{g}$ ). Under water deficit or the presence of $250 \mathrm{mM} \mathrm{NaCl}$, the color depth of the OE plants was significantly lower than that of the CK plants. In contrast, the color depth of the CRISPR plants was significantly greater than that of the CK plants (Figs. 9f-g and $10 \mathrm{f}-\mathrm{g}$ ). These results suggested that the concentration of $\mathrm{H}_{2} \mathrm{O}_{2}$ and $\mathrm{O}_{2}{ }^{-}$in the CK plants was greater than that in the OE plants but lower than that in the CRISPR plants.

The activity of NADPH oxidase (NOX) was closely related to the formation of $\mathrm{O}_{2}{ }^{-}$, the intermediate product of $\mathrm{H}_{2} \mathrm{O}_{2}$ degradation $[18,44,45]$. Therefore, we measured the concentration of $\mathrm{H}_{2} \mathrm{O}_{2}$ and NOX activity in soybean roots and leaves in accordance with the protocols of an $\mathrm{H}_{2} \mathrm{O}_{2}$ colorimetric assay kit and a NOX assay kit. The results were consistent with the staining results of $\mathrm{DAB}$ and NBT; the concentration of $\mathrm{H}_{2} \mathrm{O}_{2}$ and the NOX activity in the CK plants were $54.09 \mathrm{U} / \mathrm{g}$ and $600.95 \mathrm{U} / \mathrm{g}$, respectively, which were greater than those in the OE plants (130.77 U/ g and $1325.62 \mathrm{U} / \mathrm{g}$, respectively), and the same concentration and activity in the CK plants were lower than those in the CRISPR plants $(295.52 \mathrm{U} / \mathrm{g}$ and $2896.18 \mathrm{U} / \mathrm{g}$, respectively) under drought conditions (Fig. 9h, i). The concentration of $\mathrm{H}_{2} \mathrm{O}_{2}$ and NOX activity in the CK plants were $52.93 \mathrm{U} / \mathrm{g}$ and $641.35 \mathrm{U} / \mathrm{g}$, respectively, which were higher than those in the OE plants $(151.15 \mathrm{U} / \mathrm{g}$ and $1658.93 \mathrm{U} / \mathrm{g}$, respectively); in addition, the same concentration and activity in the CK plants were lower than those in the CRISPR plants $(276.55 \mathrm{U} / \mathrm{g}$ and $2530.05 \mathrm{U} / \mathrm{g}$, respectively) under salt conditions (Fig. 10h, i).

In addition, we stained soybean plant leaves with Trypan blue to detect cell activity under normal and stress conditions. As shown in Figs. 9e and 10e, the blue area of the OE plant leaves was obviously smaller than that of the CK plant leaves, and the CK plants were clearly smaller than the CRISPR plants under drought and salt stress conditions. No plant leaves differed under normal growth conditions (Figs. 9e and 10e). These findings suggest that the cell activity in the leaves of the CK plants is lower than that in the leaves of the OE plants but greater than that in the leaves of the CRISPR plants.

\section{Discussion}

In this study, we isolated and identified the GmMYB118 gene from 139 MYB-related transcription factors. We obtained transgenic Arabidopsis and soybean to investigate the potential function of $G m M Y B 118$. Our results indicated that $G m M Y B 118$ could improve tolerance to drought and salt stresses in Arabidopsis and soybean compared to the control lines. In present result, the encoding sequence of GmMYB118 was edited in the CRISPR hairy roots. Interestingly, the expression level of 


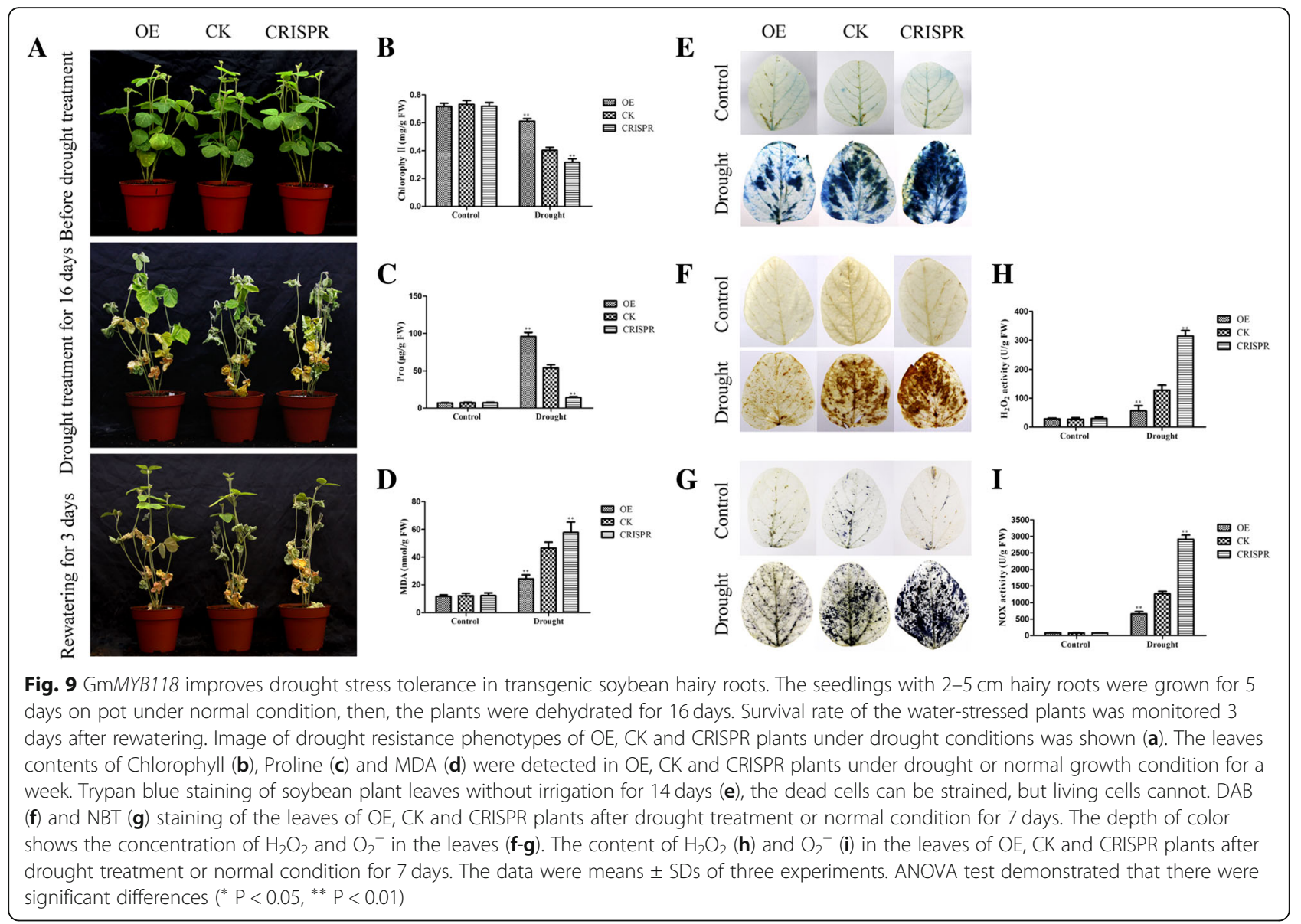

GmMYB118 in CRISPR plants was significantly lower than that in CK plants (Additional file 1: Figure S6). It suggests that the stability of mRNA may be affected after editing of $G m M Y B 118$ gene, or the decrease at the expression of GmMYB118 may be due to the removal or repair mechanism from the host itself after CRISPR editing.

Root is one of the main vegetative organs of plants, which is responsible for absorbing water and minerals dissolved in water, transporting water and minerals to stems and leaves, and storing nutrients [46]. Under the condition of drought and high salt, the root is faced with how to keep water in order to maintain the osmotic balance and to control the ion in and out of the cell membrane to maintain the ion balance, so as to increase the possibility of plant survival. In our result, the expression of GmMYB118 was the highest in the root (Fig. 3a). No previous studies have shown that the MYB-related gene is the most expressed in the root and performs some certain functions. It can be assumed that GmMYB118 can improve the osmotic balance of water and the balance of Anions and cations in the cells in the root under stresses conditions, which can directly or indirectly improve the drought resistance and salt tolerance of plants.
Previous studies showed that R2R3-MYB TFs could increase tolerance to various abiotic stresses by participating in many biochemical and physiological processes $[14,47,48]$. Few reports indicated that MYB-related TFs involved in response to abiotic stresses in plants. The MYB-related genes were mainly involved in processes, such as phytochrome regulation, flavonoid biosynthesis, hypocotyl elongation and circadian rhythm [20-23]. Currently, we have found that the expression of $G m M Y B 118$ was induced by drought, salt, heat and cold. Pi et al. reported that GmMYB173 (GmMYB118) interact with the promoter of GmCHS5 in soybean cells to regulate flavonoid biosynthesis [49]. Isoflavones has many biological functions and play an important role in the interaction between plant and environment [47, 50]. Chu et al. was reported that the green and purple leaves of sweet potatoes and the outer leaves of onion possessed higher amounts of flavonoids, and more than $85 \%$ of free radical scavenging activities were evaluated [51] It implied that GmMYB118 was involved in abiotic stresses through regulating of flavonoid biosynthesis. It also suggested that MYB-related TFs could response to abiotic stresses and the processes of flavonoid biosynthesis. 


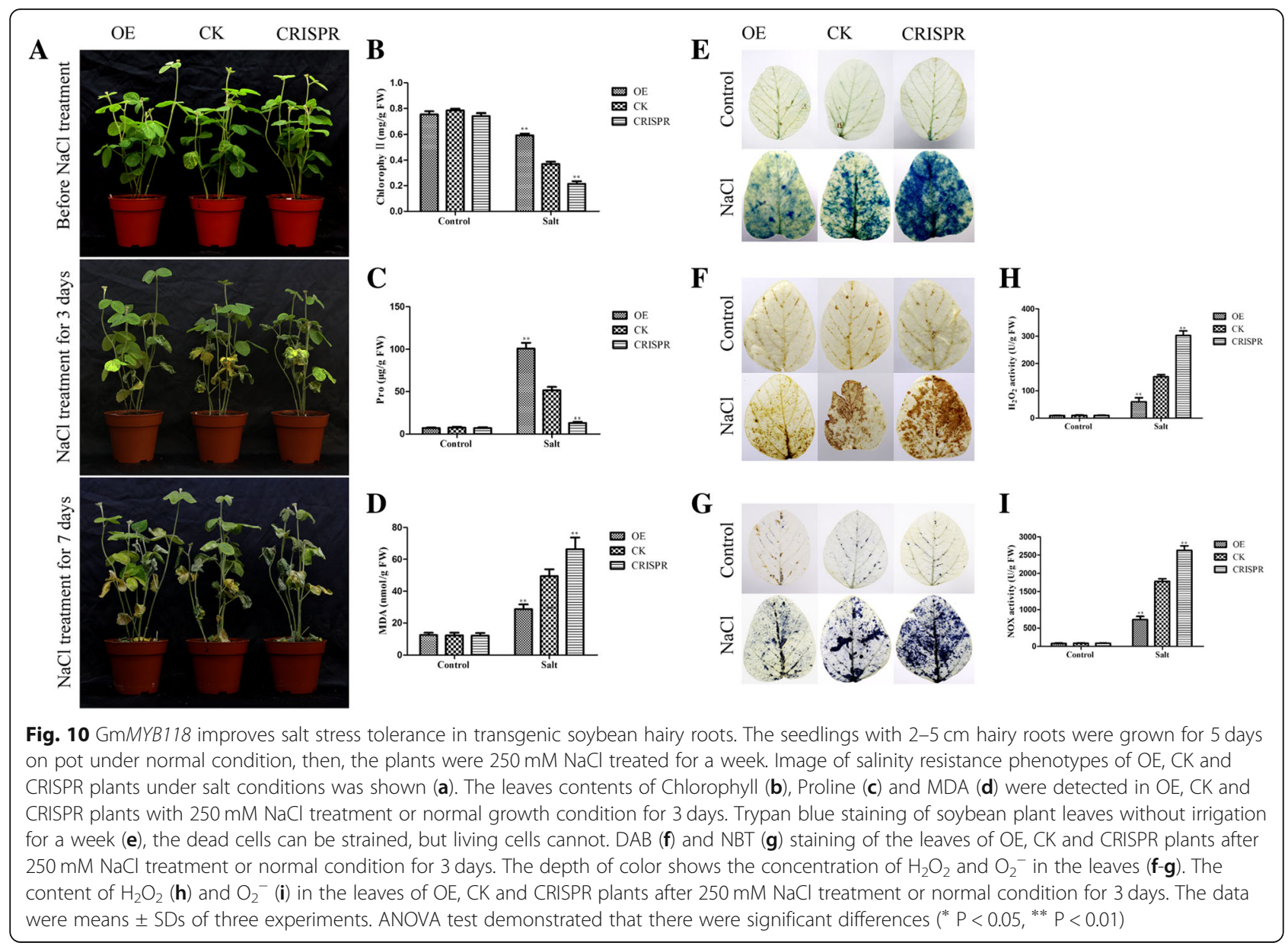

In present study, the experiments of phenotypic and molecular mechanism show that GmMYB118 improved drought resistance and salt tolerance in soybean with two approaches (OE and CRISPR) through A. rhizogenes-mediated transformation system. However, few months ago, $\mathrm{Pi}$ et al. reported that the salt-triggered phosphorylation of GmMYB173, subsequent increased in its affinity to GmCHS5 promoter and the elevated expression of GmCHS5 likely contributed to soybean salt tolerance by enhancing the accumulation of dihydroxy B-ring flavonoids [49]. Unfortunately, under drought condition, we have not found downstream genes directly regulated by GmMYB118 with some limitations of this study. In the future, it is necessary to investigate whether GmMYB118 elevate expression of GmCHS5 to enhance the accumulation of flavonoids in soybean cells under drought condition. It may reveal that whether GmMYB118 can regulate the same downstream genes to improve tolerance to drought and salt stresses, or different downstream genes to improve drought and salt tolerance. In crop science research, GmMYB118 can be used as one of the candidate genes for soybean molecular breeding in stresses resistances.

\section{Conclusion}

GmMYB118 improved tolerance to drought and salt stress by reducing the contents of ROS and MDA.

\section{Methods}

Identification of MYB-related TFs in soybean

To obtain probable candidate MYB-related TF family members, several sources such as Phytozome (http:// www.phytozome.net/) and TFDB (http://planttfdb.cbi.pku.edu.cn/) were accessed to acquire sequence data for bioinformatic analyses of soybean MYB-related TF family members. The resulting protein sequences were then examined for the presence of a MYB motif using the hidden Markov model of the SMART/Pfam tool (http://smart.embl-heidelberg.de/ and http://pfam.xfam.org/). Proteins without a MYB motif were omitted from the datasets. By using alignment and eliminating redundant sequences, we obtained 139 MYB-related TF genes, whose expression was predicted via SoyBase (http://soybase.org/sbt/).

\section{Chromosomal distribution of MYB-related genes}

Chromosomal distribution was investigated using the chromosomal loci in Phytozome. The MapInspect 
program was used to map chromosomal distributions. The deep blue bars represent the Chrs, and the Chr numbers are shown on the top of the bars. The length of the bar is not represented the size of the Chr. The numbers on the left side of the bars show the distances in megabases $(\mathrm{Mb})$ between neighboring genes.

\section{Alignment and phylogenetic analysis of MYB-related TFs Multiple alignment of the amino acid sequences was performed via ClustalX, and the alignments were manu- ally corrected. A phylogenetic tree was constructed with MEGA 6 and the NJ method, and bootstrap analysis with 1000 replicates was used to evaluate the signifi- cance of nodes [52].}

\section{Plant materials and stress treatments}

Soybean seeds (Tiefeng 8) were germinated for 15 days in pots containing vermiculite. The seedlings were then subjected to various abiotic stresses, including drought, salinity, heat, and cold stresses. For drought stress, the soybean seedlings were placed on filter paper for the induction of rapid drought for $0,1,5,12$ and $24 \mathrm{~h}$. For temperature treatments, the soybean seedlings were placed in a $4{ }^{\circ} \mathrm{C}$ or $42^{\circ} \mathrm{C}$ chamber for cold or heat treatment, respectively, for $0,1,5,12$ and $24 \mathrm{~h}$. For salt treatment, the seedlings were transferred to $250 \mathrm{mM} \mathrm{NaCl}$ solution for $0,1,5,12$ and $24 \mathrm{~h}$. All harvested seedlings were submerged immediately in liquid nitrogen and stored at $-80^{\circ} \mathrm{C}$ for RNA extraction.

Arabidopsis ecotypes Col-0 was used in this study. Seeds were germinated on $1 / 2$ MS medium with $2 \%$ sucrose, after 3 days of vernalization at $4{ }^{\circ} \mathrm{C}$, the plates containing the seeds were housed in a growth chamber that was maintained at a temperature of $22^{\circ} \mathrm{C}$, an irradiance of $40 \mu \mathrm{mol} / \mathrm{m}^{2} / \mathrm{s}^{1}$, and a photoperiod of $16 \mathrm{~h}$ light/ $8 \mathrm{~h}$ dark.

\section{RNA extraction and qRT-PCR}

Trizol reagent was used to extract total RNA in accordance with the manufacturer's protocol (TIANGEN, China), and the total RNA was treated with DNase I (TaKaRa, Japan) to remove genomic DNA contamination. qRT-PCR was completed with a PrimeScript ${ }^{\mathrm{mm}}$ RT Reagent Kit (TaKaRa, Japan) following the manufacturer's protocol. A pair of gene-specific primers was designed according to soybean MYB-related genes and stress-responsive genes in Arabidopsis via Primer Premier 5.0. The Arabidopsis and soybean actin gene were used as a control (RT-AtActin and RT-GmActin, Additional file 1: Table S1). qRT-PCR was performed with an ABI Prism 7500 real-time PCR system (ThermoFisher Scientific, USA) equipped with programs in accordance with the methods of Liu [30]. A quantitative analysis was performed using the $2^{-\Delta \Delta C T}$ method [53]. The primers used for qRT-PCR are listed in Additional file 1: Table S1.

\section{Vector construction}

The coding sequences of $G m M Y B 118$ were amplified by PCR primers (MYB118-F: ATGTCTCGCGCCTCCTC, MYB118-R: AGCAACACTAATGATGCTTTCT). Then, the restriction site (NcoI and BsTEII) in conjunction with gene-specific primers (MYB118-3301, Additional file 1: Table S2) was added to the ends of the GmMYB118 sequence. The PCR products and pCAMBIA3301 vector were digested with NcoI and BsTEII (ThermoFisher Scientific, USA), after which the products were ligated into pCAMBIA3301 under the control of the CaMV $35 \mathrm{~S}$ promoter to generate pCAMBIA3301-GmMYB118. For the CRISPR vector, sgRNA seeds of $G m M Y B 118$ were designed by CRISPR-P 2.0 (http://crispr.hzau.edu.cn), which provides web services for computer-aided design of highly efficient sgRNA that exert minimal off-target effects [54]. The sequence of sgRNA seeds was GAACAGTAT GATCTCACCGG, it was located in the first exon of the GmMYB118 gene. The restriction enzyme site (BsaI) sequences (ATTG and AAAC), respectively, was added to the end of the seed and its reverse sequence (sgRNA seeds, Additional file 1: Table S2) to obtain sgRNAs. The pUC57-GmU6 vector was digested completely with BsaI (NEB, USA); afterward, the sgRNAs was ligated into pUC57-GmU6 to obtain pUC57-GmU6-sgRNA. The primer U6-sgRNA (Additional file 1: Table S2) was used to detect whether the sequence is correct or not. The pUC57-GmU6-sgRNA and pCAMBIA3301-Cas9 vectors were digested completely with EcoRI and HindIII (ThermoFisher Scientific, USA) to obtain the fragment of GmU6-sgRNA and the vector was digested, respectively. After digestion, the fragment of GmU6 -sgRNA was cloned into the pCAMBIA3301-Cas9 vector with T4 DNA ligase (TransGene, China) to generate pCas9-GmU6-sgRNA vectors. The primer pCas9 (Additional file 1: Table S2) was used to detect whether the sequence is correct or not. All primers are listed in Additional file 1: Table S2.

\section{A. rhizogenes-mediated transformation of soybean hairy roots}

To generate transformed soybean hairy roots, the soybean cultivar Williams 82 was used for A. rhizogenes-mediated transformation [43]. Seeds were germinated under a $16 \mathrm{~h}$ light $/ 8 \mathrm{~h}$ dark photoperiod at $25^{\circ} \mathrm{C}$ in a humidity chamber. After a week, healthy plants were injected with $A$. rhizogenes strain $\mathrm{K} 599$ harboring pCAMBIA3301 (CK) or K599 harboring the construct described above (pCAMBIA3301 or pCas9-GmU6-sgRNA-construct vectors). The infected plants were then transferred to the chamber and 
kept under high humidity until hairy roots were generated at the infection site and had grown to $2-5 \mathrm{~cm}$ in length. The original main roots were removed from the $0.5 \mathrm{~cm}$ area below the infection site, then the seedlings with 2-5 $\mathrm{cm}$ hairy roots were transferred to pot for 5 days. Afterward, the plants were subjected to drought and $250 \mathrm{mM}$ $\mathrm{NaCl}$ treatment for 16 days and 7 days $[1,42]$.

\section{Promoter analysis of ten select MYB-related TFs}

The 2000 bp region upstream of the ATG start codon of the promoters of MYB family-related genes were selected to identify the cis-acting elements by submitting the promoter regions to PLACE (http://bioinformatics.psb.ugent.be/webtools/plantcare/html/). The numbers of each element were then counted manually.

\section{Trypan blue, DAB and NBT staining}

The seedlings with $2-5 \mathrm{~cm}$ hairy roots were transferred to pot for 5 days and then subjected to drought (no irrigation) for a week or $250 \mathrm{mM} \mathrm{NaCl}$ for 3 days in a growth chamber. Detached leaves from the treated seedlings were stained separately. For DAB staining, the samples were immersed in DAB solution (Solarbio, China) for $12 \mathrm{~h}$ and then in $75 \%$ ethanol for decoloring until the leaves become white. For NBT staining, the samples were immersed in NBT staining solution (Creek Huizhi, China) for $12 \mathrm{~h}$ and then in $75 \%$ ethanol [18] decoloring until the leaves become white. For Trypan blue staining, differently, the plants were subjected to drought for 16 days. The samples were immersed in 0.5\% Trypan blue (BioDee, China) solution for $12 \mathrm{~h}$ and then in $75 \%$ ethanol for decoloring until the leaves become white. Images were taken with Canon 50D (Canon, Japan) camera.

\section{Quantification of the $\mathrm{H}_{2} \mathrm{O}_{2}$ content and NOX activity}

Prior to $\mathrm{H}_{2} \mathrm{O}_{2}$ measurements, the soybean plants that transferred to pot for 5 days were subjected to drought and $250 \mathrm{mM} \mathrm{NaCl}$ stress for a week and 3 days. Afterward, the $\mathrm{H}_{2} \mathrm{O}_{2}$ content of leaves was determined in accordance with the protocol of an $\mathrm{H}_{2} \mathrm{O}_{2}$ colorimetric assay kit (Beyotime, China) [18]. Similarly, the NOX activity of leaves was determined with a NOX assay kit (Solarbio, China) in accordance with the manufacturer's protocol. All the measurements were repeated three times, and ANOVA test was used for statistical analysis.

\section{Subcellular localization assays}

The full-length cDNA sequences of GmMYB118 were fused to the $\mathrm{N}$-terminus of the $h G F P$ gene (MYB118-GFP, Additional file 1: Table S2) under the control of the CaMV $35 \mathrm{~S}$ promoter. The cDNA coding sequences of AtWRKY25 (At2g30250) that Located in the nucleus [29] were fused to the $\mathrm{N}$-terminus of the $m$ Cherry gene (WRKY25-RFP, Additional file 1: Table
S2) under the control of the CaMV 35S promoter. The recombinant plasmid of GmMYB118-GFP and AtWRKY25-RFP were cotransformed into Arabidopsis protoplasts via the PEG4000-mediated method $[18,55]$. The expression of the fusion protein was observed under dark conditions for $12 \mathrm{~h}$, and GFP and RFP was detected by laser scanning confocal microscopy (Zeiss LSM 700, Germany) [18, 30].

\section{Drought and salt stress assays of transgenic Arabidopsis plants}

To obtain EX plants, the full-length cDNA sequence of GmMYB118 was introduced into a pCAMBIA1302 plant transformation vector (MYB118-3301, Additional file 1: Table S2). Recombinant vectors were confirmed by sequencing, after which they were then transformed into Agrobacterium tumefaciens (GV3101). WT Arabidopsis thaliana (Col-0) plants were then infected with the transformed bacteria by the floral dip method [56].

The seeds of WT and EX (independent transgenic lines 4, 5 and 6) lines were disinfected with sodium hypochlorite. After 3 days of vernalization at $4{ }^{\circ} \mathrm{C}$, the plates containing the seeds were transferred to a growth chamber. Three-week-old Arabidopsis seedlings were subjected to qRT-PCR analysis of GmMYB118 gene expression in ectopic expression and WT (Col-0) plants (Additional file 1: Figure S2B). Expression of AtActin was analyzed as a loading control (Additional file 1: Table S1).

For germination assays, approximately 80 sterilized seeds of every genotype of the WT and EX plants were sown on 1/2-strength MS growth media that were supplemented with various concentrations of PEG6000 (0, 3, 6 and 9\%) (Merck, USA) or $\mathrm{NaCl}(0,75,100$ and 125 $\mathrm{mM}$ ) (XiLONG, China). The plates were housed in a growth chamber that was maintained at a temperature of $22^{\circ} \mathrm{C}$, an irradiance of $40 \mu \mathrm{mol} / \mathrm{m}^{2} / \mathrm{s}^{1}$, and a photoperiod of $16 \mathrm{~h}$ light $/ 8 \mathrm{~h}$ dark, as described previously [57, 58]. The number of germinated seeds was counted every $12 \mathrm{~h}$, and at least 80 seeds per genotype were measured.

For root growth assays, sterilized WT and EX seeds were sown on 1/2-strength MS growth media. Five-day-old seedlings were transferred to growth media that contained different concentrations of PEG6000 (0, 3, 6 and 9\%) (Merck, USA) or $\mathrm{NaCl}(0,75,100$ and $125 \mathrm{mM})$ (XiLONG, China) for a week. Images were collected after 7 days of growth, and the root lengths were evaluated via an Epson Expression 11000XL root system scanning analyzer (Epson, Japan) [57]. At least 30 seedlings per genotype were measured.

To test drought and salt tolerance at later developmental stages, three-week-old seedlings were subjected to dehydration or $250 \mathrm{mM} \mathrm{NaCl}$ for 14 days. The plant phenotypes were imaged, and the plants were counted to determine the survival rate. At least 30 seedlings were 
measured per line in each treatment, and all stress assays were performed at least three times.

\section{Heat and freezing stress assays of transgenic Arabidopsis plants}

To test the heat tolerance at the seedling stage, sterilized WT and EX seeds were sown on 1/2-strength MS growth media. Five-day-old seedlings were subjected to $37^{\circ} \mathrm{C}$ for $1 \mathrm{~h}$, allowed to recover at $22^{\circ} \mathrm{C}$ for $2 \mathrm{~h}$, and then subjected to $44^{\circ} \mathrm{C}$ for $4.5 \mathrm{~h}$ [59]. For freezing tolerance assays, 5-day-old seedlings were subjected to $-4{ }^{\circ} \mathrm{C}$

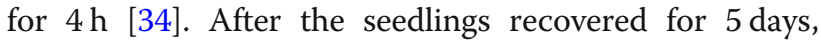
their phenotypes were imaged, and the plants were counted to determine the survival rate. At least 60 seedlings were measured per line in each treatment, and all stress assays were performed at least three times.

\section{Measurements of proline and MDA contents}

Prior to measurements, the soybean plants that transferred into pot for 5 days were subjected to drought or $250 \mathrm{mM} \mathrm{NaCl}$ stress for a week or 3 days, after which the proline content of leaves was measured as described previously [60]. Similarly, the MDA content of leaves was determined with an MDA assay kit (Comin, China) in accordance with the manufacturer's protocol. All the measurements were repeated three times, and ANOVA test was used for statistical analyses.

\section{Additional file}

Additional file 1: For disinfection of Arabidopsis thaliana seeds, the seeds of WT and EX (independent transgenic lines 4, 5 and 6) lines was disinfected with sodium hypochlorite. After 3 days of vernalization at $4^{\circ}$ $\mathrm{C}$, the plates containing the seeds was transferred to a growth chamber. For statistical methods of data, the data shown are the means \pm SDs $(n=$ 80) of three experiments. ANOVA tests demonstrated that there were significant differences $\left(* P<0.05, * *^{P}<0.01\right)$. Expression of AtActin was analyzed as a loading control in Arabidopsis (Table S1). Expression of GmActin was analyzed as a control in soybean (Table S1.) Figure S1. Quantitative gene expression of all MYB-related TF family members in soybean. The tissue expression data of quantified prediction for a diverse set of fourteen tissue types from the soybase website (http://soybase.org/ soyseq/). Figure S2. Subcellular localization and expression level of GmMYB118. GmMYB118 was localized in the nucleus in Arabidopsis mesophyll protoplasts (A). Scale bars $=20 \mu \mathrm{m}$. GmMYB118 gene expression in ectopic expression and WT (Col-0) plants (B). Figure S3. Germination rate of OE lines under PEG treatment. Images of germinating EX and WT (Col-0) seeds after $72 \mathrm{~h}$ under 0, 3, 6 and 9\% PEG6000 treatment (A). The germination of the WT and EX plants of sown on 1/2-strength MS growth media with $0 \%(B), 3 \%(C), 6 \%(D)$ and 9\% PEG6000 (E) were monitored until to $72 \mathrm{~h}$. Figure S4. Germination rate of EX lines under $\mathrm{NaCl}$ treatment. Images of germinating EX and WT (Col-0) seeds after 72 $\mathrm{h}$ under $0,75,100$ and $125 \mathrm{mM} \mathrm{NaCl}$ treatment (A). The germination of the WT and EX plants of sown on 1/2-strength MS growth media with 0 (B), 75 (C), 100 (D) and $125 \mathrm{mM} \mathrm{NaCl}$ (E) were monitored until to $72 \mathrm{~h}$. Figure S5. Targeted mutagenesis in soybean hairy roots and GUS staining. The GUS staining of transgenic hairy roots revealed a transformation efficiency of approximately 50\% (A). The target gene was amplified with specific primers and sequenced (AuGCT, China). The results show that some bases have been replaced (B). Figure S6. Expression level of GMMYB118 under drought and salt treatments. Expression level of GMMYB118 under drought (B) and salt (C) treatments was quantified by qRT-PCR assays. The expression level of GMMYB118 under normal condition was shown in Figure S6A. Table S1. Gene-specific primers used for qRT-PCR. Table S2. Primers used to construct recombinant vectors. (PDF $753 \mathrm{~kb}$ )

\section{Abbreviations}

A. rhizogenes: Agrobacterium rhizogenes; ABA: Abscisic acid; Chr: Chromosome; DAB: 3,3-diaminobenzidine; DBD: DNA-binding domain; GUS: $\beta$-glucuronidase; MDA: Malondialdehyde; NBT: Nitroblue tetrazolium; NOX: NADPH oxidase; PEG: Polyethylene glycol; qRT-PCR: Quantitative real-time PCR; ROS: Reactive oxygen species; TF: Transcription factor; WT: Wild type

\section{Acknowledgements}

We are grateful to Drs. Hui Zhang (Institute of Crop Science, Chinese Academy of Agricultural Sciences) for providing the pCAMBIA3301 vector and pUC57-GmU6-sgRNA. We thank Dr. Lijuan Qiu who comes from Crop Science, Chinese Academy of Agricultural Sciences and has provided us soybean seeds (Tiefeng 8).

\section{Funding}

The design and data collection of this study were supported by the National Transgenic Key Project of the Ministry of Agriculture of China (2018ZX0800909B). Analysis and interpretation of data in this research were supported by the Funding Project for Beijing Advanced Innovation Center for Food Nutrition and Human Health, the Open Research Fund Program of Beijing Key Lab of Plant Resource Research and Development, Beijing Technology and Business University. The manuscript was written with financial support from the Introduction of Wheat Germplasm with Fusarium Crown Rot Resistance and Molecular Marker-Assisted Breeding (1639630D).

\section{Availability of data and materials}

The accession number of the maker gene located in the nucleus is At2g30250. The accession number of the GmMYB118 gene is GLYMA_17G094400.

\section{Authors' contributions}

ZSX coordinated the project, conceived and designed experiments, and edited the manuscript; YTD performed the experiments and wrote the first draft of the manuscript; MJZ, CTW and YG conducted the bioinformatic work and performed related experiments; YXW,YWL and YBZ provided analytical tools and analyzed the data; MC and JC contributed valuable discussion; and YZM coordinated the project. All authors have read and approved the final manuscript.

\section{Ethics approval and consent to participate}

Not applicable.

\section{Consent for publication \\ Not applicable.}

\section{Competing interests}

The authors declare that they have no competing interests.

\section{Publisher's Note}

Springer Nature remains neutral with regard to jurisdictional claims in published maps and institutional affiliations.

\section{Author details}

${ }^{1}$ Institute of Crop Sciences, Chinese Academy of Agricultural Sciences (CAAS)/National Key Facility for Crop Gene Resources and Genetic Improvement, Key Laboratory of Biology and Genetic Improvement of Triticeae Crops, Ministry of Agriculture, Beijing 100081, China. ${ }^{2}$ Beijing Advanced Innovation Center for Food Nutrition and Human Health/Beijing Key Lab of Plant Resource Research and Development, Beijing Technology and Business University, Beijing 100048, China. ${ }^{3}$ Shijiazhuang Academy of Agricultural and Forestry Sciences, Research Center of Wheat Engineering Technology of Hebei, Shijiazhuang 050041, Hebei, China. ${ }^{4}$ Institute of Genetics and Physiology, Hebei Academy of Agriculture and Forestry Sciences/Plant Genetic Engineering Center of Hebei Province, Shijiazhuang 050051, Hebei, China. 


\section{Received: 22 August 2018 Accepted: 21 November 2018} Published online: 03 December 2018

\section{References}

1. Wang F, Chen HW, Li QT, Wei W, Li W, Zhang WK, Ma B, Bi YD, Lai YC, Liu XL, et al. GmWRKY27 interacts with GmMYB174 to reduce expression of GmNAC29 for stress tolerance in soybean plants. Plant J. 2015;83(2):224-36.

2. Yamaguchi-Shinozaki K, Shinozaki K. Transcriptional regulatory networks in cellular responses and tolerance to dehydration and cold stresses. Annu Rev Plant Biol. 2006;57:781-803.

3. Xu ZS, Chen M, Li LC, Ma YZ. Functions of the ERF transcription factor family in plants. Botany. 2008:86(9):969-77.

4. $\quad \mathrm{Xu} Z \mathrm{ZS}$, Chen M, Li LC, Ma YZ. Functions and application of the AP2/ERF transcription factor family in crop improvement. J Integr Plant Biol. 2011:53(7):570-85

5. Yanhui $C$, Xiaoyuan $Y$, Kun $H$, Meihua $L$, Jigang $L$, Zhaofeng $G$, Zhiqiang $L$, Yunfei Z, Xiaoxiao W, Xiaoming Q, et al. The MYB transcription factor superfamily of Arabidopsis: expression analysis and phylogenetic comparison with the rice MYB family. Plant Mol Biol. 2006;60(1):107-24.

6. Zhao Y, Tian X, Wang F, Zhang L, Xin M, Hu Z, Yao Y, Ni Z, Sun Q, Peng H. Characterization of wheat MYB genes responsive to high temperatures. BMC Plant Biol. 2017;17(1):208.

7. Chu S, Wang J, Zhu Y, Liu S, Zhou X, Zhang H, Wang CE, Yang W, Tian Z, Cheng H, et al. An R2R3-type MYB transcription factor, GmMYB29, regulates isoflavone biosynthesis in soybean. PLoS Genet. 2017;13(5):e1006770.

8. Liu L. The roles of MYB transcription factors on plant defense responses and its molecular mechanism. Hereditas (Beijing). 2008;30(10):1265-71.

9. Martin C, PazAres J. MYB transcription factors in plants. Trends Genet. 1997; 13(2):67-73.

10. Bian SM, Jin DH, Li RH, Xie X, Gao GL, Sun WK, Li YJ, Zhai LL, Li XY. Genome-Wide Analysis of CCA1-Like Proteins in Soybean and Functional Characterization of GmMYB138a. Int J Mol Sci. 2017;18(10). https://doi.org/ 10.3390/ijms18102040.

11. Wang F, Suo Y, Wei H, Li M, Xie C, Wang L, Chen X, Zhang Z. Identification and characterization of 40 isolated Rehmannia glutinosa MYB family genes and their expression profiles in response to shading and continuous cropping. Int J Mol Sci. 2015;16(7):15009-30.

12. Xiong H, Li J, Liu P, Duan J, Zhao Y, Guo X, Li Y, Zhang H, Ali J, Li Z. Overexpression of OsMYB48-1, a novel MYB-related transcription factor, enhances drought and salinity tolerance in rice. PLoS One. 2014;9(3):e92913.

13. Baldoni $E$, Genga A, Cominelli E. Plant MYB transcription factors: their role in drought response mechanisms. Int J Mol Sci. 2015;16(7):15811-51.

14. Dubos C, Stracke R, Grotewold E, Weisshaar B, Martin C, Lepiniec L. MYB transcription factors in Arabidopsis. Trends Plant Sci. 2010;15(10):573-81.

15. Feng G, Burleigh JG, Braun EL, Mei W, Barbazuk WB. Evolution of the 3RMYB gene family in plants. Genome Biol Evol. 2017;9(4):1013-29.

16. Zhang L, Zhao G, Xia C, Jia J, Liu X, Kong X. Overexpression of a wheat MYB transcription factor gene, TaMYB56-B, enhances tolerances to freezing and salt stresses in transgenic Arabidopsis. Gene. 2012;505(1):100-7.

17. Xu R, Wang Y, Zheng H, Lu W, Wu C, Huang J, Yan K, Yang G, Zheng C. Saltinduced transcription factor MYB74 is regulated by the RNA-directed DNA methylation pathway in Arabidopsis. J Exp Bot. 2015;66(19):5997-6008.

18. Wang N, Zhang WX, Qin MY, Li S, Qiao M, Liu ZH, Xiang FN. Drought tolerance conferred in soybean (Glycine max. L) by GmMYB84, a novel R2R3-MYB transcription factor. Plant Cell Physiol. 2017;58(10):1764-76.

19. Chen N, Yang Q, Pan L, Chi X, Chen M, Hu D, Yang Z, Wang T, Wang M, Yu S. Identification of 30 MYB transcription factor genes and analysis of their expression during abiotic stress in peanut (Arachis hypogaea L.). Gene. 2014:533(1):332-45.

20. Kobayashi S, Ishimaru M, Hiraoka K, Honda C. Myb-related genes of the Kyoho grape ( Vitis labruscana) regulate anthocyanin biosynthesis. Planta. 2002;215(6):924-33.

21. Lee MM, Schiefelbein J. WEREWOLF, a MYB-related protein in Arabidopsis, is a position-dependent regulator of epidermal cell patterning. Cell. 1999;99(5):473-83.

22. Noda K, Glover BJ, Linstead P, Martin C. Flower colour intensity depends on specialized cell shape controlled by a Myb-related transcription factor. Nature. 1994;369(6482):661-4.

23. Nguyen NH, Lee H. MYB-related transcription factors function as regulators of the circadian clock and anthocyanin biosynthesis in Arabidopsis. Plant Signal Behav. 2016;11(3). https://doi.org/10.1080/15592324.2016.1139278.
24. Glover BJ, Perez-Rodriguez M, Martin C. Development of several epidermal cell types can be specified by the same MYB-related plant transcription factor. Development. 1998;125(17):3497-508.

25. Yi J, Derynck MR, Li X, Telmer P, Marsolais F, Dhaubhadel S. A single-repeat MYB transcription factor, GmMYB176, regulates CHS8 gene expression and affects isoflavonoid biosynthesis in soybean. Plant J. 2010;62(6):1019-34.

26. Urao T, Noji M, YamaguchiShinozaki K, Shinozaki K. A transcriptional activation domain of ATMYB2, a drought-inducible Arabidopsis Myb-related protein. Plant J. 1996;10(6):1145-8.

27. Schmutz J, Cannon SB, Schlueter J, Ma J, Mitros T, Nelson W, Hyten DL, Song Q, Thelen JJ, Cheng J, et al. Genome sequence of the palaeopolyploid soybean. Nature. 2010;463(7278):178-83.

28. Du H, Wang YB, Xie Y, Liang Z, Jiang SJ, Zhang SS, Huang YB, Tang YX. Genome-wide identification and evolutionary and expression analyses of MYB-related genes in land plants. DNA Res. 2013;20(5):437-48.

29. Jiang YQ, Deyholos MK. Functional characterization of Arabidopsis NaClinducible WRKY25 and WRKY33 transcription factors in abiotic stresses. Plant Mol Biol. 2009;69(1-2):91-105.

30. Liu P, Xu ZS, Lu PP, Hu D, Chen M, Li LC, Ma YZ. A wheat PI4K gene whose product possesses threonine autophophorylation activity confers tolerance to drought and salt in Arabidopsis. J Exp Bot. 2013;64(10):2915-27.

31. Riechmann JL, Heard J, Martin G, Reuber L, Jiang CZ, Keddie J, Adam L, Pineda O, Ratcliffe OJ, Samaha RR, et al. Arabidopsis transcription factors: genome-wide comparative analysis among eukaryotes. Science. 2000; 290(5499):2105-10.

32. Flowers TJ. Improving crop salt tolerance. J Exp Bot. 2004;55(396):307-19.

33. Cheng L, Li X, Huang X, Ma T, Liang Y, Ma X, Peng X, Jia J, Chen S, Chen Y, et al. Overexpression of sheepgrass R1-MYB transcription factor LcMYB1 confers salt tolerance in transgenic Arabidopsis. Plant Physiol Biochem. 2013;70:252-60

34. Liu Q, Kasuga M, Sakuma Y, Abe H, Miura S, Yamaguchi-Shinozaki K, Shinozaki K, Baek D. Two transcription factors, DREB1 and DREB2, with an EREBP/AP2 DNA binding domain separate two cellular signal transduction pathways in drought- and low-temperature-responsive gene expression, respectively, in Arabidopsis. Plant Cell. 1998;10(8):1391-406.

35. Yamaguchi-Shinozakiaib K, and, Shinozaki K. A Nove1 cis-acting element in an Arabidopsis gene 1s Involved in responsiveness to drought, Lowqemperature, or High-Salt Stress. Plant Cell. 1994, 6(2):251-264.

36. Kurkela S, Franck M. Cloning and characterization of a cold- and ABAinducible Arabidopsis gene. Plant Mol Biol. 1990;15(1):137-44.

37. Kurkela S, Borg-Franck M. Structure and expression of kin2, one of two cold- and ABA-induced genes of Arabidopsis thaliana. Plant Mol Biol. 1992;19(4):689-92.

38. Harshavardhan VT, Van Son L, Seiler C, Junker A, Weigelt-Fischer K, Klukas C, Altmann T, Sreenivasulu N, Baumlein H, Kuhlmann M. AtRD22 and AtUSPL1, members of the plant-specific BURP domain family involved in Arabidopsis thaliana drought tolerance. PLoS One. 2014;9(10):e110065.

39. Lang V, Palva ET. The expression of a Rab-related gene, rab18, is induced by abscisic acid during the cold acclimation process of Arabidopsis thaliana (L.) Heynh. Plant Mol Biol. 1992;20(5):951-62.

40. Hoeren FU, Dolferus R, Wu Y, Peacock WJ, Dennis ES. Evidence for a role for AtMYB2 in the induction of the Arabidopsis alcohol dehydrogenase gene (ADH1) by low oxygen. Genetics. 1998;149(2):479-90.

41. Ruggiero B, Koiwa H, Manabe Y, Quist TM, Inan G, Saccardo F, Joly RJ, Hasegawa PM, Bressan RA, Maggio A. Uncoupling the effects of abscisic acid on plant growth and water relations. Analysis of sto1/nced3, an abscisic acid-deficient but salt stress-tolerant mutant in arabidopsis. Plant Physiol. 2004;136(2):3134-47.

42. Sun $X, H u Z$, Chen $R$, Jiang $Q$, Song $G$, Zhang $H, X i$ Y. Targeted mutagenesis in soybean using the CRISPR-Cas9 system. Sci Rep. 2015;5:10342.

43. Kereszt A, Li DX, Indrasumunar A, Nguyen CDT, Nontachaiyapoom S, Kinkema M, Gresshoff PM. Agrobacterium rhizogenes - mediated transformation of soybean to study root biology. Nat Protoc. 2007;2(4):948-52.

44. Mittler R, Vanderauwera S, Gollery M, Van Breusegem F. Reactive oxygen gene network of plants. Trends Plant Sci. 2004;9(10):490-8.

45. Foreman J, Demidchik V, Bothwell JHF, Mylona P, Miedema H, Torres MA, Linstead P, Costa S, Brownlee C, Jones JDG, et al. Reactive oxygen species produced by NADPH oxidase regulate plant cell growth. Nature. 2003;422(6930):442-6.

46. Schachtman DP, Goodger JQD. Chemical root to shoot signaling under drought. Trends Plant Sci. 2008;13(6):281-7.

47. Czemmel S, Heppel SC, Bogs J. R2R3 MYB transcription factors: key regulators of the flavonoid biosynthetic pathway in grapevine. Protoplasma. 2012;249(Suppl 2):S109-18 
48. Du H, Yang SS, Liang Z, Feng BR, Liu L, Huang YB, Tang YX. Genome-wide analysis of the MYB transcription factor superfamily in soybean. BMC Plant Biol. 2012;12:106

49. Pi E, Zhu C, Fan W, Huang Y, Qu L, Li Y, Zhao Q, Ding F, Qiu L, Wang H, et al. Quantitative Phosphoproteomic and Metabonomic Analyses Reveal GmMYB173 Optimizes Flavonoid Metabolism in Soybean under Salt Stress. MCP Papers in Press. 2018;17(5):mcp.RA117.000417.

50. Koes RE, Quattrocchio F, Mol JNM. The flavonoid biosynthetic pathway in plants: function and evolution. Bioessays. 1994;16(2):123-32.

51. Chu YH, Chang CL, Hsiafen H. Flavonoid content of several vegetables and their antioxidant activity. J Sci Food Agric. 2000;80(5):561-6.

52. Tamura K, Stecher G, Peterson D, Filipski A, Kumar S. MEGA6: molecular evolutionary genetics analysis version 6.0. Mol Biol Evol. 2013;30(12):2725-9.

53. Le DT, Nishiyama R, Watanabe Y, Mochida K, Yamaguchi-Shinozaki K, Shinozaki K, Tran LS. Genome-wide expression profiling of soybean twocomponent system genes in soybean root and shoot tissues under dehydration stress. DNA Res. 2011;18(1):17-29.

54. Lei Y, Lu L, Liu HY, Li S, Xing F, Chen LL. CRISPR-P: a web tool for synthetic single-guide RNA design of CRISPR-system in plants. Mol Plant. 2014;7(9):1494-6.

55. He GH, Xu JY, Wang YX, Liu JM, Li PS, Chen M, Ma YZ, Xu ZS. Droughtresponsive WRKY transcription factor genes TaWRKY1 and TaWRKY33 from wheat confer drought and/or heat resistance in Arabidopsis. BMC Plant Biol. 2016;16:116.

56. Clough SJ, Bent AF. Floral dip: a simplified method for agrobacteriummediated transformation of Arabidopsis thaliana. Plant J. 1998;16(6):735-43.

57. Zhao SP, Xu ZS, Zheng WJ, Zhao W, Wang YX, Yu TF, Chen M, Zhou YB, Min $\mathrm{DH}, \mathrm{Ma} \mathrm{YZ}$, et al. Genome-wide analysis of the RAV family in soybean and functional identification of GmRAV-03 involvement in salt and drought stresses and exogenous ABA treatment. Front Plant Sci. 2017;8:905.

58. Feng $\mathrm{CZ}$, Chen $\mathrm{Y}$, Wang $\mathrm{C}$, Kong $\mathrm{YH}, \mathrm{Wu}$ WH, Chen YF. Arabidopsis RAV transcription factor, phosphorylated by SnRK2 kinases, regulates the expression of $A B|3, A B| 4$, and $A B \mid 5$ during seed germination and early seedling development. Plant J. 2014;80(4):654-68.

59. Li SX, Liu JX, Liu ZY, Li XR, Wu FJ, He YK. HEAT-INDUCED TAS1 TARGET1 mediates Thermotolerance via HEAT STRESS TRANSCRIPTION FACTOR A1adirected pathways in Arabidopsis. Plant Cell. 2014;26(4):1764-80

60. Bu QY, Lv TX, Shen H, Luong P, Wang J, Wang ZY, Huang ZG, Xiao LT, Engineer C, Kim TH, et al. Regulation of drought tolerance by the F-box protein MAX2 in Arabidopsis(1 [C][W][OPEN]). Plant Physiol. 2014;164(1):424-39.

Ready to submit your research? Choose BMC and benefit from:

- fast, convenient online submission

- thorough peer review by experienced researchers in your field

- rapid publication on acceptance

- support for research data, including large and complex data types

- gold Open Access which fosters wider collaboration and increased citations

- maximum visibility for your research: over $100 \mathrm{M}$ website views per year

At $\mathrm{BMC}$, research is always in progress.

Learn more biomedcentral.com/submissions 